\title{
Planar master integrals for the two-loop light-fermion electroweak corrections to Higgs plus jet production
}

\author{
Matteo Becchetti, ${ }^{a, b}$ Roberto Bonciani, ${ }^{a, b}$ Valerio Casconi, ${ }^{a, b}$ Vittorio Del Duca ${ }^{c, 1}$ \\ and Francesco Moriello ${ }^{c}$ \\ ${ }^{a}$ Sapienza - Università di Roma, Dipartimento di Fisica, \\ Piazzale Aldo Moro 5, 00185, Rome, Italy \\ ${ }^{b}$ INFN Sezione di Roma, \\ Piazzale Aldo Moro 2, 00185, Rome, Italy \\ ${ }^{c}$ ETH Zürich, Institut für theoretische Physik, \\ Wolfgang-Pauli str. 27, 8093, Zürich, Switzerland \\ E-mail: matteo.becchetti@roma1.infn.it, \\ roberto.bonciani@roma1.infn.it, valerio.casconi@roma1.infn.it, \\ delducav@itp.phys.ethz.ch, fmoriello@phys.ethz.ch
}

ABStRaCT: We present the analytic calculation of the planar master integrals which contribute to compute the two-loop light-fermion electroweak corrections to the production of a Higgs boson in association with a jet in gluon-gluon fusion. The complete dependence on the electroweak-boson mass is retained. The master integrals are evaluated by means of the differential equations method and the analytic results are expressed in terms of multiple polylogarithms up to weight four.

KEYwords: NLO Computations

ARXiv EPrint: 1810.05138

\footnotetext{
${ }^{1}$ On leave from INFN, Laboratori Nazionali di Frascati, Italy.
} 


\section{Contents}

1 Introduction 1

2 Notations 3

3 The system of differential equations 4

$\begin{array}{ll}3.1 & \text { The alphabet }\end{array}$

$\begin{array}{lll}4 & \text { The master integrals } & 7\end{array}$

$\begin{array}{lll}5 & \text { Conclusions } & 7\end{array}$

$\begin{array}{lrr}\text { A Routing for the pre-canonical master integrals } & 8\end{array}$

B The canonical basis $\quad 10$

\section{Introduction}

Since its discovery in 2012 [1, 2], the study of the Higgs boson has been one of the dominant topics in the physics programme of the Large Hadron Collider (LHC). The Higgs signals provide a strong test of the Standard Model (SM) and can be a probe of New Physics (NP), in case a deviation from the SM behaviour of the Higgs properties will be revealed [3-6].

The major production channel of the Higgs boson at the LHC is gluon-gluon fusion. Since the Higgs boson does not couple directly to gluons, the coupling is mediated by a heavy-quark loop. Thus, the leading-order (LO) production requires the evaluation of one-loop amplitudes, the production at the next-to-leading order (NLO) accuracy requires the evaluation of two-loop amplitudes, and so forth. So far, in the full theory, i.e. with a complete dependence on the heavy-quark mass, Higgs production via gluon-gluon fusion is known at NLO [7, 8] (see also [9-12]). However, in the limit in which the heavy-quark mass is much heavier than the other scales in the process, the computations are made more tractable by using the Higgs Effective Field Theory (HEFT), i.e. by replacing the loopmediated Higgs-gluon coupling by a tree-level effective coupling. That lowers by one loop the amplitudes to be computed. In the HEFT, Higgs production via gluon-gluon fusion is known at $\mathrm{N}^{3} \mathrm{LO}$ [13-17], whose accuracy has reached the $5 \%$ level [18].

In order to have access to a detailed study of the Higgs properties, precise theoretical predictions for more exclusive observables are needed. Of particular importance, for a detailed study of the SM structure of the loop-mediated Higgs-gluon coupling and of possible NP contributions to it, is the prediction of the transverse momentum distribution of a Higgs boson at high $p_{T}$ [19-31].

The transverse momentum distribution of the Higgs boson in gluon-gluon fusion is known in the HEFT at NNLO [32-36]. While this approximation is satisfactory in the 
region in which the Higgs $p_{T}$ is smaller than the top-quark mass, in the high- $p_{T}$ region it is known to give rise to predictions that can differ considerably from the ones in which the top-quark mass is treated exactly [37]. In the full theory, the production of a Higgs boson in association with a jet is known only at LO [38, 39]. Several approximations exist to Higgs plus one jet production at NLO in the full theory: $(i)$ a numerical computation [40], which includes, though, only the dependence on the top-quark mass, while neglecting the bottom-quark mass; (ii) computations in the high $p_{T}$ region [41, 42], based on the two-loop amplitudes for Higgs plus three partons, computed in the limit when the Higgs transverse momentum is larger than the top-quark mass, $p_{T} \gg m_{t}$ [43].; (iii) computations in the intermediate $p_{T}$ region, where it is relevant to include also the top-bottom interference [44, 45], which is estimated by interfering a top-quark loop computed in the HEFT with a bottom-quark loop computed as an expansion in a small bottom-quark mass [46, 47].

The exact evaluation of the Higgs plus one jet production at NLO in the full theory, i.e. retaining the complete dependence on the mass of the heavy quark that runs into the loops, requires to compute the two-loop four-point amplitudes for Higgs plus three partons, where one of the loops is a heavy-quark loop, which involve elliptic iterated integrals. So far, only the planar contributions have been computed [48].

In addition, although the Higgs boson does not couple directly to massless fermions, its coupling to light fermions may be mediated by an electroweak-boson loop. In particular, the Higgs boson may also couple to gluons via a double loop, made by an electroweakboson loop and a light-quark loop $[49,50]$. This production mode makes up the bulk of the two-loop electroweak corrections to Higgs production via gluon-gluon fusion [51-53] and increases by about $5 \%$ the $\mathrm{LO}$ gluon-fusion cross section and by about $2 \%$ the $\mathrm{N}^{3} \mathrm{LO}$ cross section [18].

The NLO QCD corrections to the two-loop light-quark contribution to Higgs production via gluon-gluon fusion consist of the three-loop mixed QCD-electroweak corrections to Higgs production [54, 55], and of the two-loop four-point amplitudes for Higgs plus three partons, where one loop is an electroweak-boson loop and the other is a light-quark loop. These two-loop four-point amplitudes are presently unknown. Two approximated evaluations of the NLO QCD corrections have been computed: $(a)$ the full corrections in the un-physical limit where the Higgs mass is much smaller than the electroweak-boson mass [56]; (b) a computation in which the virtual part is treated exactly [55], but the real radiation is included in the soft-gluon approximation [57].

In this paper, we present the analytic computation of the planar master integrals (MIs) relevant to the evaluation of the two-loop four-point amplitudes for Higgs plus three gluons, where one loop is an electroweak-boson loop and the other is a light-quark loop. They contribute to the mixed QCD-electroweak light-fermion corrections to the production of a Higgs boson with an additional jet, as well as to the real radiation of the NLO QCD corrections to the two-loop light-quark contribution to Higgs production via gluon-gluon fusion.

We performed the reduction to the MIs using the computer softwares FIRE5 [58] and LiteRed [59], ${ }^{1}$ which implement Integration-by-parts identities (IBPs) [63-65] and Lorentzinvariance [66] identities, obtaining 48 MIs. Apart from the two-point functions, these MIs

\footnotetext{
${ }^{1}$ Other public available softwares [60-62] for the IBPs reduction exist.
} 
are new and presented in this paper for the first time. They are analitically computed using the differential equations method [67-71]. This method has proved to be very efficient for the computation of the MIs needed for higher-order corrections in the SM. In particular, we adopt the canonical basis approach [71-77] to the solution of the system of differential equations, which is expressed in terms of Chen-iterated integrals [78] represented as Goncharov multiple polylogarithms [79-81] (GPLs) up to weight four. The solutions are evaluated numerically using the software GiNaC $[82,83]$ and tested numerically against the software FIESTA [84] in the Euclidean and Minkowski regions of the phase space. We find agreement in both regions.

The analytic results presented in this paper are given as ancillary files uploaded with the arXiv submission. ${ }^{2}$

The paper is structured as follows. In the section 2 we give our notations and we describe the kinematic of the processes studied. In section 3 we describe briefly the method of differential equations and the canonical basis approach, moreover we give the alphabet associated to the solution. In section 4 we present the canonical basis and the transformation among the MIs in canonical form and the pre-canonical ones. Finally, in section 5 we present our conclusion.

\section{Notations}

In this paper we consider the partonic processes $g g \rightarrow H g, g q \rightarrow q H, q \bar{q} \rightarrow g H$, and the crossed channels $H \rightarrow g g g$ and $H \rightarrow q \bar{q} g$. The external momenta corresponding either to gluons or to quarks are on their mass-shell $p_{i}^{2}=0$, while the external Higgs momentum $p_{4}^{2}$ is regarded as a variable. Therefore, we appoach the solution of the master integrals (MIs) of the topology as a three-scale problem, where, apart from the Higgs momentum $p_{4}^{2}$, the other two variables are the Mandelstam variables

$$
s=\left(p_{1}+p_{2}\right)^{2}, \quad t=\left(p_{1}+p_{3}\right)^{2} .
$$

For later convenience we define the dimensionless variabiles $x, y, z$ such that

$$
x=-\frac{s}{4 m_{B}^{2}}, \quad y=-\frac{t}{4 m_{B}^{2}}, \quad z=-\frac{p_{4}^{2}}{4 m_{B}^{2}},
$$

where $m_{B}^{2}$ is the squared mass of the internal Electroweak boson ( $W$ or $Z$ ).

The physical phase-space region of the kinematic invariants (2.2) is

$$
x<0, \quad y<0, \quad z<x+y,
$$

for the decay channel, while for the production channel is

$$
x<z<0, y>0, \quad z>x+y .
$$

The planar corrections can be computed considering the 7-denominator topology shown in figure 1 . The MIs of the topology are defined by the two-loop dimensionally regularized

\footnotetext{
${ }^{2}$ The ancillary files are in text format and the results, expressed as GPLs, are written in GiNac format.
} 


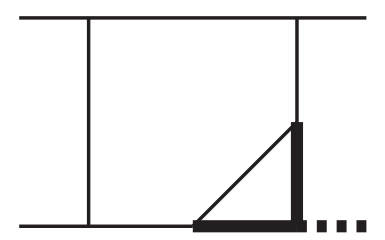

Figure 1. Planar seven-denominator topology.

integrals

$$
\int \mathcal{D}^{d} k_{1} \mathcal{D}^{d} k_{2} \frac{D_{8}^{a_{8}} D_{9}^{a_{9}}}{D_{1}^{a_{1}} D_{2}^{a_{2}} D_{3}^{a_{3}} D_{4}^{a_{4}} D_{5}^{a_{5}} D_{6}^{a_{6}} D_{7}^{a_{7}}}
$$

where $d=4-2 \epsilon, a_{i}$ with $i=1, \ldots, 7$ are integer numbers, while $a_{8}$ and $a_{9}$ are natural numbers and the normalization is such that

$$
\mathcal{D}^{d} k_{i}=\frac{d^{d} k_{i}}{i \pi^{\frac{d}{2}}} e^{\epsilon \gamma_{E}}\left(\frac{m_{B}^{2}}{\mu^{2}}\right)^{\epsilon} .
$$

The $D_{i}, i=1, \ldots, 7$, are the denominators involved, while $D_{8}, D_{9}$ the numerators. They belong to the following set:

$$
\begin{aligned}
& \left\{-k_{1}^{2},-\left(k_{1}-k_{2}\right)^{2},-\left(p_{1}+k_{1}\right)^{2},-\left(p_{1}+p_{2}+k_{1}\right)^{2},-\left(p_{3}-k_{1}\right)^{2},\right. \\
& \left.\quad-\left(p_{1}+p_{2}+k_{2}\right)^{2}+m_{B}^{2},-\left(p_{3}-k_{2}\right)^{2}+m_{B}^{2},-\left(p_{3}+k_{1}-k_{2}\right)^{2},-\left(k_{2}+p_{2}\right)^{2}\right\} .
\end{aligned}
$$

The number of MIs for this topology is 48, considering the different channels and crossings. Apart from two-point functions, ${ }^{3}$ the MIs presented in this paper are new.

\section{The system of differential equations}

The analytic computation of the master integrals is performed by using the differential equations method [67-70] applied to a canonical basis for the MIs [71, 72]. In order to find the canonical basis several approaches exist [71-75]. We adopt the semi-algorithmic approach described in [76, 77]. The canonical basis $\vec{f}(\vec{x}, \epsilon)$ satisfies a system of first order partial linear differential equations with respect to the kinematic invariants $\vec{x}$,

$$
\frac{\partial \vec{f}(\vec{x}, \epsilon)}{\partial x_{i}}=\epsilon A_{i}(\vec{x}) \vec{f}(\vec{x}, \epsilon)
$$

where $i \in\{1, \ldots, n\}, n$ is the number kinematic invariants and $A_{i}(\vec{x})$ is the set of matrices defining the differential equations. The linear system of partial differential equations, eq. (3.1), is equivalent to the following differential form,

$$
d \vec{f}(\vec{x}, \epsilon)=\epsilon d \tilde{A}(\vec{x}) \vec{f}(\vec{x}, \epsilon),
$$

where the matrix elements of $\tilde{A}(\vec{x})$ are $\mathbb{Q}$-linear combinations of logarithms, with

$$
\frac{\partial \tilde{A}(\vec{x})}{\partial x_{i}}=A_{i}(\vec{x}) .
$$

\footnotetext{
${ }^{3}$ The two-point functions can be found for instance in $[85,86]$.
} 
The set of linearly indipendent arguments of the logarithms is called the alphabet of the solution, and its elements are called letters. The matrices $A_{i}(\vec{x})$ satisfy the integrability condition

$$
\frac{\partial A_{j}}{\partial x_{i}}-\frac{\partial A_{i}}{\partial x_{j}}=0, \quad\left[A_{i}, A_{j}\right]=0
$$

where $\left[A_{i}, A_{j}\right]=A_{i} A_{j}-A_{j} A_{i}$. The solution of the differential equations (3.2) can be formally written as a path ordered exponential:

$$
\vec{f}(\vec{x}, \epsilon)=\mathbb{P} \exp \left(\epsilon \int_{\gamma} d \tilde{A}(\vec{x})\right) \vec{f}\left(\vec{x}_{0}, \epsilon\right),
$$

where $\mathbb{P}$ stands for the path-ordering operator, $\gamma$ is a path in the space of kinematic invariants and $\vec{f}\left(\vec{x}_{0}, \epsilon\right)$ is a vector of boundary conditions. In practice we are interested in a solution around $\epsilon=0$. By series expanding $\vec{f}(\vec{x}, \epsilon)$,

$$
\vec{f}(\vec{x}, \epsilon)=\sum_{k=0}^{\infty} \epsilon^{k} \vec{f}^{(k)}(\vec{x})
$$

and by parametrizing the integration contour with $t \in[0,1]$, the solution, eq. (3.5), translates to iterated integrals [78]:

$$
\vec{f}(x)=\vec{f}^{(0)}\left(\vec{x}_{0}\right)+\sum_{k=1}^{\infty} \epsilon^{k} \sum_{j=1}^{k} \int_{0}^{1} d t_{1} \frac{\partial \tilde{A}\left(t_{1}\right)}{\partial t_{1}} \int_{0}^{t_{1}} d t_{2} \frac{\partial \tilde{A}\left(t_{2}\right)}{\partial t_{2}} \ldots \int_{0}^{t_{j-1}} d t_{j} \frac{\partial \tilde{A}\left(t_{j}\right)}{\partial t_{j}} \vec{f}^{(k-j)}\left(\vec{x}_{0}\right) .
$$

In general, the alphabet letters depend algebraically on the kinematic invariants $x_{i}$. However, for the problem under consideration, it is possible to perform a variable change such that the alphabet depends only rationally on the new variables. This implies that the solution eq. (3.7) can be directly expressed in terms of Goncharov multiple polylogarithms (GPLs) [79, 87], defined recursively as,

$$
G\left(\alpha_{1}, \ldots, \alpha_{n} ; z\right)=\int_{0}^{z} \frac{d t}{t-\alpha_{1}} G\left(\alpha_{2}, \ldots, \alpha_{n} ; t\right) .
$$

The recursion ends when $n=0$ where we conventionally set

$$
G(; z) \equiv 1
$$

Moreover, in order to deal with the divergency at the basepoint 0 when $\alpha_{n}=0$, one defines:

$$
G(\overrightarrow{0} ; z) \equiv \frac{1}{n !} \log (z)^{n}
$$

We remark that also when the alphabet letters are not rational functions it is often possible to find a representation of the solution in terms of polylogarithmic functions. One starts from the symbol [88] of the solution, which is obtained from the differential equations matrix $\tilde{A}(\vec{x})$ and the $\epsilon^{0}$ order of the boundary conditions, by the following recursive formula,

$$
\mathcal{S}\left(f_{n}^{(i)}(\vec{x})\right)=\sum_{m} \mathcal{S}\left(f_{m}^{(i-1)}(\vec{x})\right) \otimes \mathcal{S}\left(\tilde{A}_{n m}(\vec{x})\right) .
$$

The corresponding polylogarithmic expressions are found by using the algorithm of $[88,89]$, and its algebraic generalisation [48]. 


\subsection{The alphabet}

The system of differential equations depends originally on rational functions of $x$ and $y$ and on the following square root

$$
\sqrt{z(1+z)} \text {. }
$$

Exploiting for instance the methods described in [77], it is possible to rationalize the square root (3.12) by means of the change of variables

$$
z \rightarrow \frac{w^{2}}{1+2 w} .
$$

It is then straightforward to define the differential equations with respect to $x, y, w$ defined by the matrices $A_{x}(x, y, w), A_{y}(x, y, w), A_{w}(x, y, w)$ respectively and solve them by using the following iterative formula,

$$
\begin{aligned}
f_{j}^{(i)}(x, y, w)= & \sum_{k}\left[\int_{x_{0}, y_{0}, w_{0}}^{x, y_{0}, w_{0}} A_{x, j k}(x, y, w) f_{k}^{(i-1)}(x, y, w) d x\right. \\
& +\int_{x, y_{0}, w_{0}}^{x, y, w_{0}} A_{y, j k}(x, y, w) f_{k}^{(i-1)}(x, y, w) d y \\
& \left.+\int_{x, y, w_{0}}^{x, y, w} A_{w, j k}(x, y, w) f_{k}^{(i-1)}(x, y, w) d w\right] \\
& +f_{j}^{(i)}\left(x_{0}, y_{0}, w_{0}\right),
\end{aligned}
$$

where we denoted by, e.g., $A_{x, j k}(x, y, w)$ the matrix element of the matrix $A_{x}(x, y, w)$ and $x_{0}, y_{0}, w_{0}$ is a set of boundary points that in general depend on the master integral (see next section). The recursion above can be directly solved in terms of GPLs by factorizing the matrix elements with respect to the integration variable. By performing the factorization we obtain the (inverse) GPLs integration kernels, $\left(x-x_{k}\right),\left(y-y_{k}\right),\left(w-w_{k}\right)$, with

$$
\begin{aligned}
x_{k} \in & \left\{0,-\frac{1}{4}, \frac{1}{4}\right\}, \\
y_{k} \in & \left\{0,-\frac{1}{4}, \frac{1}{4},-x,-\frac{x}{4 x+1}\right\}, \\
w_{k} \in & \left\{0,-\frac{1}{2},-1, \frac{1}{4}(-1-i \sqrt{3}), \frac{1}{4}(-1+i \sqrt{3}), 2 x,-\frac{2 x}{4 x+1}, x-\sqrt{x^{2}+x}\right. \\
& x+\sqrt{x^{2}+x}, \frac{1}{4}\left(-1+4 x-\sqrt{16 x^{2}+8 x-3}\right), \frac{1}{4}\left(-1+4 x+\sqrt{16 x^{2}+8 x-3}\right), \\
& 2 y,-\frac{2 y}{4 y+1}, y-\sqrt{y^{2}+y}, y+\sqrt{y^{2}+y}, \frac{1}{4}\left(-1+4 y-\sqrt{16 y^{2}+8 y-3}\right) \\
& \frac{1}{4}\left(-1+4 y+\sqrt{16 y^{2}+8 y-3}\right), x+y-\sqrt{x^{2}+2 x y+x+y^{2}+y} \\
& \left.x+y+\sqrt{x^{2}+2 x y+x+y^{2}+y}, \frac{-x-y}{2 y}, \frac{-x-y}{2 x}\right\}
\end{aligned}
$$

that correspond to the arguments of the GPLs of the solution. 


\section{The master integrals}

The set of 48 MIs, which we refer as $\mathcal{T}_{i}, i \in\{1, \ldots, 48\}$, is shown in figure 2 . The corresponding canonical basis elements $f_{i}(\vec{x})$ are defined as linear combinations of pre-canonical integrals with algebraic prefactors of the Mandelstam invariants and the space-time regulator. Their definition is provided in appendix B.

The boundary conditions for the canonical master integrals $f_{3}, \ldots, f_{6}, f_{8}, \ldots, f_{24}, f_{26}$, $\ldots, f_{36}, f_{38}, f_{40}, \ldots, f_{48}$ are fixed in the point $s=t=p_{4}^{2}=0$, which is a regular point for the previous canonical master integrals, instead, the master integrals $f_{1}, f_{2}, f_{7}, f_{25}$ are divergent in $s=t=p_{4}^{2}=0$, but they are product of known one-loop master integrals. Finally, for the master integrals $f_{37}, f_{39}$ we fix the boundary conditions, respectively, in the regular points $\left(s=0, t=m_{B}^{2}, p_{4}^{2}=0\right)$ and $\left(s=m_{B}^{2}, t=0, p_{4}^{2}=0\right)$.

The solution is obtained in the un-physical region defined by:

$$
x \geq 0, \quad y \geq 0, \quad w \geq 0 .
$$

Since we are able to express all the canonical integrals $f_{1} \ldots f_{48}$ in terms of GPLs up to weight 4 , the numerical check in all the regions of the phase space is straightforward. For the numerical evaluation of the masters we use the software GiNaC $[82,83]$. The analytic continuation in the Minkowski region is perfomed (numerically) adding a small imaginary part to the squared c.m. energy, $s+i 0^{+}$. The Minkowski physical regions are given, in terms of the variables $x, y$ and $w$, by:

$$
\begin{array}{llll}
\text { H decay: } & x<0, & y<0, & \frac{w^{2}}{1+2 w}<x+y, \\
\mathrm{H}+\text { jet: } & x<\frac{w^{2}}{1+2 w}<0, & y>0, & \frac{w^{2}}{1+2 w}>x+y .
\end{array}
$$

where no branch cuts are present. We checked different points of the phase space against FIESTA [84], finding complete agreement.

\section{Conclusions}

In this paper we have computed analytically the planar master integrals relevant to the evaluation of the two-loop four-point amplitudes for Higgs plus three gluons, where one loop is an electroweak-boson loop and the other is a light-quark loop. Those amplitudes contribute to the mixed QCD-electroweak light-fermion corrections to the production of a Higgs boson with an additional jet, as well as to the real radiation of the NLO QCD corrections to the two-loop light-quark contribution to Higgs production via gluon-gluon fusion.

The master integrals are evaluated with the differential equations method applied to a canonical set of basis integrals. Since the alphabet of the solution depends on a single square root, it is possible to find a variable change such that the matrices associated to the system of differential equations can be expressed in terms of rational functions. This allows a direct integration of the differential equations in terms of generalized polylogarithms up to weight 4 . The expression of the master integrals in terms of generalized polylogarithms is quite flexible and can be evaluated numerically in a fast and precise way. 


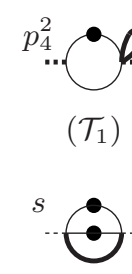

( $\left.\mathcal{T}_{9}\right)$

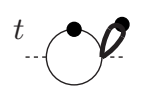

$\left(\mathcal{T}_{2}\right)$

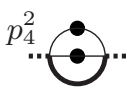

$\left(\mathcal{T}_{3}\right)$

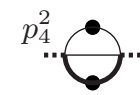

$\left(\mathcal{T}_{4}\right)$

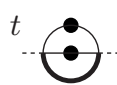

$\left(\mathcal{T}_{5}\right)$

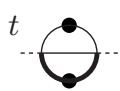

$\left(\mathcal{T}_{6}\right)$

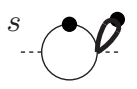

$\left(\mathcal{T}_{7}\right)$

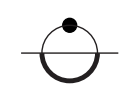

$\left(\mathcal{T}_{8}\right)$

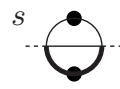

$\left(\mathcal{T}_{10}\right)$

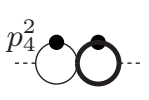

$\left(\mathcal{T}_{11}\right)$

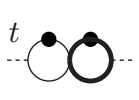

$\left(\mathcal{T}_{12}\right)$

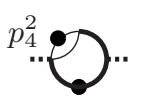

$\left(\mathcal{T}_{13}\right)$

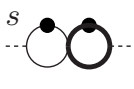

$\left(\mathcal{T}_{14}\right)$

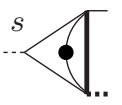

$\left(\mathcal{T}_{21}\right)$

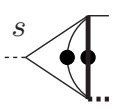

$\left(\mathcal{T}_{22}\right)$

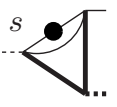

$\left(\mathcal{T}_{15}\right)$

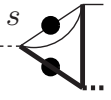

$\left(\mathcal{T}_{16}\right)$

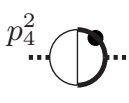

$\left(\mathcal{T}_{23}\right)$

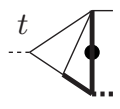

$\left(\mathcal{T}_{30}\right)$

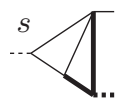

$\left(\mathcal{T}_{31}\right)$

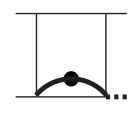

$\left(\mathcal{T}_{38}\right)$

$\left(\mathcal{T}_{37}\right)$

$\left(\mathcal{T}_{36}\right)$

$\left(\mathcal{T}_{35}\right)$

L.

$\left(\mathcal{T}_{43}\right)$

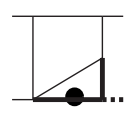

$\left(\mathcal{T}_{44}\right)$

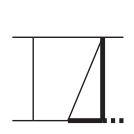

$\left(\mathcal{T}_{45}\right)$

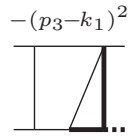

$\left(\mathcal{T}_{46}\right)$

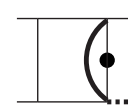

$\left(\mathcal{T}_{39}\right)$

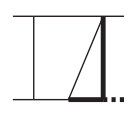

$\left(\mathcal{T}_{47}\right)$

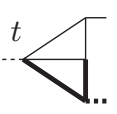

$\left(\mathcal{T}_{24}\right)$

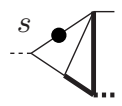

$\left(\mathcal{T}_{32}\right)$

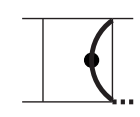

$\left(\mathcal{T}_{40}\right)$

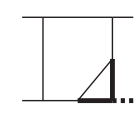

$\left(\mathcal{T}_{48}\right)$

Figure 2. Master integrals in pre-canonical form. Internal plain thin lines represent massless propagators, while thick lines represent the vector-boson propagator. External plain thin lines represent massless particles on their mass-shell. External dashed thin lines represent the dependence on $s, t$, or $m_{H}^{2}$. The external dashed thick line represents the Higgs on its mass-shell.

\section{Acknowledgments}

MB and VC thank the Institut für Theoretische Physik of the ETH Zürich for the hospitality and the COST (European Cooperation in Science and Technology) Action CA16201 PARTICLEFACE for the support, during the early stages of this work.

\section{A Routing for the pre-canonical master integrals}

In this appendix we give the expression for the pre-canonical master integrals in the form of eq. (2.5).

$$
\begin{array}{ll}
\mathcal{T}_{1}=\int \mathcal{D}^{d} k_{1} \mathcal{D}^{d} k_{2} \frac{1}{D_{4}^{2} D_{5} D_{7}^{2}}, & \mathcal{T}_{2}=\int \mathcal{D}^{d} k_{1} \mathcal{D}^{d} k_{2} \frac{1}{D_{3}^{2} D_{5} D_{7}^{2}}, \\
\mathcal{T}_{3}=\int \mathcal{D}^{d} k_{1} \mathcal{D}^{d} k_{2} \frac{1}{D_{2}^{2} D_{5}^{2} D_{6}}, & \mathcal{T}_{4}=\int \mathcal{D}^{d} k_{1} \mathcal{D}^{d} k_{2} \frac{1}{D_{2}^{2} D_{5} D_{6}^{2}}, \\
\mathcal{T}_{5}=\int \mathcal{D}^{d} k_{1} \mathcal{D}^{d} k_{2} \frac{1}{D_{2}^{2} D_{3}^{2} D_{7}}, & \mathcal{T}_{6}=\int \mathcal{D}^{d} k_{1} \mathcal{D}^{d} k_{2} \frac{1}{D_{2}^{2} D_{3} D_{7}^{2}},
\end{array}
$$




$$
\begin{aligned}
& \mathcal{T}_{7}=\int \mathcal{D}^{d} k_{1} \mathcal{D}^{d} k_{2} \frac{1}{D_{1}^{2} D_{4} D_{7}^{2}}, \\
& \mathcal{T}_{9}=\int \mathcal{D}^{d} k_{1} \mathcal{D}^{d} k_{2} \frac{1}{D_{1}^{2} D_{2}^{2} D_{6}}, \\
& \mathcal{T}_{11}=\int \mathcal{D}^{d} k_{1} \mathcal{D}^{d} k_{2} \frac{1}{D_{4}^{2} D_{5} D_{6} D_{7}^{2}}, \\
& \mathcal{T}_{13}=\int \mathcal{D}^{d} k_{1} \mathcal{D}^{d} k_{2} \frac{1}{D_{2}^{2} D_{5} D_{6}^{2} D_{7}}, \\
& \mathcal{T}_{15}=\int \mathcal{D}^{d} k_{1} \mathcal{D}^{d} k_{2} \frac{1}{D_{1}^{2} D_{2} D_{6} D_{7}}, \\
& \mathcal{T}_{17}=\int \mathcal{D}^{d} k_{1} \mathcal{D}^{d} k_{2} \frac{1}{D_{2}^{2} D_{3} D_{6} D_{7}}, \\
& \mathcal{T}_{19}=\int \mathcal{D}^{d} k_{1} \mathcal{D}^{d} k_{2} \frac{1}{D_{2}^{2} D_{3} D_{5} D_{6}}, \\
& \mathcal{T}_{21}=\int \mathcal{D}^{d} k_{1} \mathcal{D}^{d} k_{2} \frac{1}{D_{1} D_{2}^{2} D_{4} D_{7}}, \\
& \mathcal{T}_{23}=\int \mathcal{D}^{d} k_{1} \mathcal{D}^{d} k_{2} \frac{1}{D_{2} D_{4} D_{5} D_{6} D_{7}^{2}}, \\
& \mathcal{T}_{25}=\int \mathcal{D}^{d} k_{1} \mathcal{D}^{d} k_{2} \frac{1}{D_{1} D_{3} D_{4} D_{5} D_{7}^{2}}, \\
& \mathcal{T}_{27}=\int \mathcal{D}^{d} k_{1} \mathcal{D}^{d} k_{2} \frac{1}{D_{2} D_{3} D_{5} D_{6} D_{7}}, \\
& \mathcal{T}_{29}=\int \mathcal{D}^{d} k_{1} \mathcal{D}^{d} k_{2} \frac{1}{D_{2} D_{3} D_{5}^{2} D_{6}^{2} D_{7}}, \\
& \mathcal{T}_{31}=\int \mathcal{D}^{d} k_{1} \mathcal{D}^{d} k_{2} \frac{1}{D_{1} D_{2} D_{4} D_{6} D_{7}}, \\
& \mathcal{T}_{33}=\int \mathcal{D}^{d} k_{1} \mathcal{D}^{d} k_{2} \frac{1}{D_{1} D_{2} D_{4}^{2} D_{6} D_{7}^{2}}, \\
& \mathcal{T}_{35}=\int \mathcal{D}^{d} k_{1} \mathcal{D}^{d} k_{2} \frac{1}{D_{1} D_{2} D_{3} D_{6} D_{7}}, \\
& \mathcal{T}_{37}=\int \mathcal{D}^{d} k_{1} \mathcal{D}^{d} k_{2} \frac{1}{D_{1} D_{2}^{2} D_{3} D_{5} D_{6}}, \\
& \mathcal{T}_{39}=\int \mathcal{D}^{d} k_{1} \mathcal{D}^{d} k_{2} \frac{1}{D_{1} D_{2}^{2} D_{3} D_{4} D_{7}}, \\
& \mathcal{T}_{41}=\int \mathcal{D}^{d} k_{1} \mathcal{D}^{d} k_{2} \frac{1}{D_{1} D_{3} D_{4} D_{5} D_{6} D_{7}^{2}}, \\
& \mathcal{T}_{43}=\int \mathcal{D}^{d} k_{1} \mathcal{D}^{d} k_{2} \frac{D_{4}}{D_{1} D_{2} D_{3} D_{5} D_{6} D_{7}}, \\
& \mathcal{T}_{45}=\int \mathcal{D}^{d} k_{1} \mathcal{D}^{d} k_{2} \frac{1}{D_{1} D_{2} D_{3} D_{4} D_{6} D_{7}}, \\
& \mathcal{T}_{47}=\int \mathcal{D}^{d} k_{1} \mathcal{D}^{d} k_{2} \frac{1}{D_{1} D_{2} D_{3} D_{4} D_{6} D_{7}^{2}},
\end{aligned}
$$

$$
\begin{aligned}
& \mathcal{T}_{8}=\int \mathcal{D}^{d} k_{1} \mathcal{D}^{d} k_{2} \frac{1}{D_{1}^{2} D_{2} D_{7}}, \\
& \mathcal{T}_{10}=\int \mathcal{D}^{d} k_{1} \mathcal{D}^{d} k_{2} \frac{1}{D_{1}^{2} D_{2} D_{6}^{2}}, \\
& \mathcal{T}_{12}=\int \mathcal{D}^{d} k_{1} \mathcal{D}^{d} k_{2} \frac{1}{D_{3}^{2} D_{5} D_{6}^{2} D_{7}}, \\
& \mathcal{T}_{14}=\int \mathcal{D}^{d} k_{1} \mathcal{D}^{d} k_{2} \frac{1}{D_{1}^{2} D_{4} D_{6} D_{7}^{2}}, \\
& \mathcal{T}_{16}=\int \mathcal{D}^{d} k_{1} \mathcal{D}^{d} k_{2} \frac{1}{D_{1}^{2} D_{2} D_{6}^{2} D_{7}}, \\
& \mathcal{T}_{18}=\int \mathcal{D}^{d} k_{1} \mathcal{D}^{d} k_{2} \frac{1}{D_{2}^{2} D_{3} D_{6} D_{7}^{2}}, \\
& \mathcal{T}_{20}=\int \mathcal{D}^{d} k_{1} \mathcal{D}^{d} k_{2} \frac{1}{D_{2}^{2} D_{3} D_{5} D_{6}^{2}}, \\
& \mathcal{T}_{22}=\int \mathcal{D}^{d} k_{1} \mathcal{D}^{d} k_{2} \frac{1}{D_{1} D_{2}^{2} D_{4} D_{7}^{2}}, \\
& \mathcal{T}_{24}=\int \mathcal{D}^{d} k_{1} \mathcal{D}^{d} k_{2} \frac{1}{D_{2} D_{3} D_{4} D_{6} D_{7}}, \\
& \mathcal{T}_{26}=\int \mathcal{D}^{d} k_{1} \mathcal{D}^{d} k_{2} \frac{1}{D_{1} D_{2} D_{5} D_{6} D_{7}}, \\
& \mathcal{T}_{28}=\int \mathcal{D}^{d} k_{1} \mathcal{D}^{d} k_{2} \frac{1}{D_{2}^{2} D_{3} D_{5} D_{6} D_{7}}, \\
& \mathcal{T}_{30}=\int \mathcal{D}^{d} k_{1} \mathcal{D}^{d} k_{2} \frac{1}{D_{2} D_{3} D_{5} D_{6}^{2} D_{7}}, \\
& \mathcal{T}_{32}=\int \mathcal{D}^{d} k_{1} \mathcal{D}^{d} k_{2} \frac{1}{D_{1}^{2} D_{2} D_{4} D_{6} D_{7}}, \\
& \mathcal{T}_{34}=\int \mathcal{D}^{d} k_{1} \mathcal{D}^{d} k_{2} \frac{1}{D_{1} D_{2} D_{4} D_{6} D_{7}^{2}}, \\
& \mathcal{T}_{36}=\int \mathcal{D}^{d} k_{1} \mathcal{D}^{d} k_{2} \frac{1}{D_{1} D_{2}^{2} D_{3} D_{6} D_{7}}, \\
& \mathcal{T}_{38}=\int \mathcal{D}^{d} k_{1} \mathcal{D}^{d} k_{2} \frac{1}{D_{1} D_{2} D_{3} D_{5} D_{6}^{2}}, \\
& \mathcal{T}_{40}=\int \mathcal{D}^{d} k_{1} \mathcal{D}^{d} k_{2} \frac{1}{D_{1} D_{2} D_{3} D_{4} D_{7}^{2}}, \\
& \mathcal{T}_{42}=\int \mathcal{D}^{d} k_{1} \mathcal{D}^{d} k_{2} \frac{1}{D_{1} D_{2} D_{3} D_{5} D_{6} D_{7}}, \\
& \mathcal{T}_{44}=\int \mathcal{D}^{d} k_{1} \mathcal{D}^{d} k_{2} \frac{1}{D_{1} D_{2} D_{3} D_{5} D_{6}^{2} D_{7}}, \\
& \mathcal{T}_{46}=\int \mathcal{D}^{d} k_{1} \mathcal{D}^{d} k_{2} \frac{D_{5}}{D_{1} D_{2} D_{3} D_{4} D_{6} D_{7}}, \\
& \mathcal{T}_{48}=\int \mathcal{D}^{d} k_{1} \mathcal{D}^{d} k_{2} \frac{1}{D_{1} D_{2} D_{3} D_{4} D_{5} D_{6} D_{7}} .
\end{aligned}
$$




\section{B The canonical basis}

$$
\begin{aligned}
& f_{1}=\epsilon^{2} p_{4}^{2} \mathcal{T}_{1}, \\
& f_{2}=\epsilon^{2} t \mathcal{T}_{2}, \\
& f_{3}=\epsilon^{2}\left(m_{B}^{2}-p_{4}^{2}\right) \mathcal{T}_{3}+2 m_{B}^{2} \epsilon^{2} \mathcal{T}_{4}, \\
& f_{4}=\epsilon^{2} p_{4}^{2} \mathcal{T}_{4}, \\
& f_{5}=-2 m_{B}^{2} \epsilon^{2} \mathcal{T}_{6}+\epsilon^{2}\left(t-m_{B}^{2}\right) \mathcal{T}_{5} \text {, } \\
& f_{6}=\epsilon^{2} t \mathcal{T}_{6}, \\
& f_{7}=\epsilon^{2} s \mathcal{T}_{7}, \\
& f_{8}=\epsilon^{2}(\epsilon-1) \mathcal{T}_{8}, \\
& f_{9}=\epsilon^{2}\left(s-m_{B}^{2}\right) \mathcal{T}_{9}+\epsilon^{2}\left(s-2 m_{B}^{2}\right) \mathcal{T}_{10}, \\
& f_{10}=\epsilon^{2} s \mathcal{T}_{10}, \\
& f_{11}=\epsilon^{2} \sqrt{\left(p_{4}^{2}\right)^{3}} \sqrt{4 m_{B}^{2}-p_{4}^{2}} \mathcal{T}_{11}, \\
& f_{12}=\epsilon^{2} \sqrt{p_{4}^{2}} \sqrt{4 m_{B}^{2}-p_{4}^{2}} t \mathcal{T}_{12}, \\
& f_{13}=\epsilon^{2} \sqrt{p_{4}^{2}} \sqrt{4 m_{B}^{2}-p_{4}^{2}} m_{B}^{2} \mathcal{T}_{13}+\epsilon^{2}\left(m_{B}^{2}-p_{4}^{2}\right) \frac{\sqrt{4 m_{B}^{2}-p_{4}^{2}}}{4 \sqrt{p_{4}^{2}}} \mathcal{T}_{3} \\
& +\epsilon^{2}\left(m_{B}^{2}-p_{4}^{2}\right) \frac{4 m_{B}^{2}-p_{4}^{2}}{4 \sqrt{p_{4}^{2}}} \mathcal{T}_{4}-\epsilon^{2}(\epsilon-1) \frac{\sqrt{4 m_{B}^{2}-p_{4}^{2}}}{2 \sqrt{p_{4}^{2}}} \mathcal{T}_{8} \\
& f_{14}=\epsilon^{2} \sqrt{4 m_{B}^{2}-p_{4}^{2}} \sqrt{p_{4}^{2}} s \mathcal{T}_{14}, \\
& f_{15}=\epsilon^{3}\left(s-p_{4}^{2}\right) \mathcal{T}_{15} \text {, } \\
& f_{16}=\epsilon^{2} \frac{\sqrt{4 m_{B}^{2}-p_{4}^{2}} \sqrt{p_{4}^{2}}\left(m_{B}^{2} p_{4}^{2}-p_{4}^{2} s+s^{2}\right)}{2 s-p_{4}^{2}} \mathcal{T}_{16}+\epsilon^{2}(\epsilon-1) \frac{\sqrt{4 m_{B}^{2}-p_{4}^{2}} \sqrt{p_{4}^{2}}}{2 p_{4}^{2}-4 s} \mathcal{T}_{8} \\
& +\frac{\sqrt{4 m_{B}^{2}-p_{4}^{2}} \sqrt{p_{4}^{2}}\left(s-m_{B}^{2}\right)}{2\left(2 p_{4}^{2}-4 s\right)} \mathcal{T}_{9}-3 \epsilon^{3} \frac{\sqrt{4 m_{B}^{2}-p_{4}^{2}} \sqrt{p_{4}^{2}}\left(s-p_{4}^{2}\right)}{2 p_{4}^{2}-4 s} \mathcal{T}_{15}, \\
& f_{17}=\epsilon^{3}\left(t-p_{4}^{2}\right) \mathcal{T}_{17} \text {, } \\
& f_{18}=\epsilon^{2} \frac{\sqrt{4 m_{B}^{2}-p_{4}^{2}} \sqrt{p_{4}^{2}}\left(m_{B}^{2} p_{4}^{2}-p_{4}^{2} t+t^{2}\right)}{-p_{4}^{2}+2 t} \mathcal{T}_{18}-3 \epsilon^{3} \frac{\sqrt{4 m_{B}^{2}-p_{4}^{2}} \sqrt{p_{4}^{2}}\left(t-p_{4}^{2}\right)}{2 p_{4}^{2}-4 t} \mathcal{T}_{17} \\
& +\epsilon^{2}(\epsilon-1) \frac{\sqrt{4 m_{B}^{2}-p_{4}^{2}} \sqrt{p_{4}^{2}}}{2 p_{4}^{2}-4 t} \mathcal{T}_{8}+\epsilon^{2} \frac{\sqrt{4 m_{B}^{2}-p_{4}^{2}} \sqrt{p_{4}^{2}}\left(t-m_{B}^{2}\right)}{2\left(2 p_{4}^{2}-4 t\right)} \mathcal{T}_{5} \\
& +\epsilon^{2} \frac{\sqrt{4 m_{B}^{2}-p_{4}^{2}} \sqrt{p_{4}^{2}} t\left(t-m_{B}^{2}\right)}{2\left(p_{4}^{2} t-t^{2}\right)} \mathcal{T}_{6}, \\
& f_{19}=\epsilon^{3}\left(t-p_{4}^{2}\right) \mathcal{T}_{19},
\end{aligned}
$$




$$
\begin{aligned}
& f_{20}=\epsilon^{2} \frac{m_{B}^{2}\left(t-p_{4}^{2}\right)\left(m_{B}^{2}-p_{4}^{2}+t\right)}{-2 m_{B}^{2}-p_{4}^{2}+t} \mathcal{T}_{20}+\epsilon^{3} \frac{\left(p_{4}^{2}-t\right)\left(-4 m_{B}^{2}+p_{4}^{2}-t\right)}{2 m_{B}^{2}+p_{4}^{2}-t} \mathcal{T}_{19} \\
& +\epsilon^{2} \frac{\left(p_{4}^{2}-t\right) t}{2 m_{B}^{2}+p_{4}^{2}-t} \mathcal{T}_{2}-\epsilon^{2} \frac{2 m_{B}^{2}\left(m_{B}^{2}-p_{4}^{2}\right)}{-2 m_{B}^{2}-p_{4}^{2}+t} \mathcal{T}_{3}-\epsilon^{2} \frac{2 m_{B}^{2}\left(2 m_{B}^{2}+p_{4}^{2}\right)}{-2 m_{B}^{2}-p_{4}^{2}+t} \mathcal{T}_{4}, \\
& f_{21}=\epsilon^{3}\left(s-p_{4}^{2}\right) \mathcal{T}_{21} \text {, } \\
& f_{22}=\frac{\epsilon^{2} m_{B}^{2}\left(s-p_{4}^{2}\right)\left(m_{B}^{2}-p_{4}^{2}+s\right)}{-2 m_{B}^{2}-p_{4}^{2}+s} \mathcal{T}_{22}+\frac{\epsilon^{3}\left(p_{4}^{2}-s\right)\left(-4 m_{B}^{2}+p_{4}^{2}-s\right)}{2 m_{B}^{2}+p_{4}^{2}-s} \mathcal{T}_{21} \\
& -\frac{2 \epsilon^{2} m_{B}^{2}\left(m_{B}^{2}-p_{4}^{2}\right)}{-2 m_{B}^{2}-p_{4}^{2}+s} \mathcal{T}_{3}-\frac{2 \epsilon^{2} m_{B}^{2}\left(2 m_{B}^{2}+p_{4}^{2}\right)}{-2 m_{B}^{2}-p_{4}^{2}+s} \mathcal{T}_{4}+\frac{2 \epsilon^{2} m_{B}^{2} s}{-2 m_{B}^{2}-p_{4}^{2}+s} \mathcal{T}_{7}, \\
& f_{23}=\epsilon^{2}(-1+2 \epsilon) m_{B}^{2} p_{4}^{2} \mathcal{T}_{23}+\epsilon^{2} p_{4}^{4} \mathcal{T}_{11}-\epsilon^{2} m_{B}^{2} p_{4}^{2} \mathcal{T}_{13}, \\
& f_{24}=\epsilon^{4}\left(t-p_{4}^{2}\right) \mathcal{T}_{24}, \\
& f_{25}=\epsilon^{3} s t \mathcal{T}_{25} \text {, } \\
& f_{26}=\epsilon^{4}\left(s-p_{4}^{2}\right) \mathcal{T}_{26}, \\
& f_{27}=\epsilon^{4}\left(t-p_{4}^{2}\right) \mathcal{T}_{27}, \\
& f_{28}=\epsilon^{3} m_{B}^{2}\left(t-p_{4}^{2}\right) \mathcal{T}_{28}, \\
& f_{29}=\epsilon^{2} m_{B}^{2} p_{4}^{2} t \mathcal{T}_{29}+2 \epsilon^{4} t \mathcal{T}_{27}+\frac{1}{2} m_{B}^{2} \epsilon^{3}\left(p_{4}^{2}-t\right) \mathcal{T}_{28}+\frac{1}{2} p_{4}^{2} \epsilon^{3}\left(p_{4}^{2}+t\right) \mathcal{T}_{30} \\
& +\frac{\epsilon^{2}\left(-4 m_{B}^{2}+p_{4}^{2}\right) t}{2 m_{B}^{2}+p_{4}^{2}-t} \mathcal{T}_{2}-\frac{\epsilon^{2}\left(4 m_{B}^{4}-5 m_{B}^{2} p_{4}^{2}+p_{4}^{4}\right)}{-2 m_{B}^{2}-p_{4}^{2}+t} \mathcal{T}_{3}-\frac{\epsilon^{2}\left(8 m_{B}^{4}+2 m_{B}^{2} p_{4}^{2}-p_{4}^{4}\right)}{-2 m_{B}^{2}-p_{4}^{2}+t} \mathcal{T}_{4} \\
& +\frac{\epsilon^{2}\left(m_{B}^{2}-t\right) t}{2\left(p_{4}^{2}-2 t\right)} \mathcal{T}_{5}+\frac{\epsilon^{2} t\left(m_{B}^{2}-p_{4}^{2}+t\right)}{p_{4}^{2}-2 t} \mathcal{T}_{6}-\frac{(\epsilon-1) \epsilon^{2}\left(p_{4}^{2}-t\right)}{p_{4}^{2}-2 t} \mathcal{T}_{8}+p_{4}^{2} t \epsilon^{2} \mathcal{T}_{12} \\
& +m_{B}^{2} p_{4}^{2} \epsilon^{2} \mathcal{T}_{13}-\frac{\epsilon^{3}\left(3 p_{4}^{4}-5 p_{4}^{2} t+t^{2}\right)}{p_{4}^{2}-2 t} \mathcal{T}_{17}+\frac{p_{4}^{2} \epsilon^{2}\left(m_{B}^{2} p_{4}^{2}+t\left(t-p_{4}^{2}\right)\right)}{p_{4}^{2}-2 t} \mathcal{T}_{18} \\
& -\frac{2 \epsilon^{3}\left(3 p_{4}^{2}-2 t\right)\left(m_{B}^{2}-p_{4}^{2}+t\right)}{2 m_{B}^{2}+p_{4}^{2}-t} \mathcal{T}_{19}+\frac{\epsilon^{2} m_{B}^{2}\left(3 p_{4}^{2}-2 t\right)\left(m_{B}^{2}-p_{4}^{2}+t\right)}{2 m_{B}^{2}+p_{4}^{2}-t} \mathcal{T}_{20}, \\
& f_{30}=\epsilon^{3} \sqrt{p_{4}^{2}} \sqrt{4 m_{B}^{2}-p_{4}^{2}}\left(t-p_{4}^{2}\right) \mathcal{T}_{30} \\
& f_{31}=\epsilon^{4}\left(s-p_{4}^{2}\right) \mathcal{T}_{31}, \\
& f_{32}=\epsilon^{3} s\left(s-p_{4}^{2}\right) T_{32}, \\
& f_{33}=\epsilon^{2} m_{B}^{2} p_{4}^{2} s \mathcal{T}_{33}+2 \epsilon^{4}\left(p_{4}^{2}+s\right) \mathcal{T}_{31}+\epsilon^{3} s\left(s-p_{4}^{2}\right) \mathcal{T}_{32}+\epsilon^{3} p_{4}^{2}\left(p_{4}^{2}+s\right) \mathcal{T}_{34} \\
& +\frac{2 \epsilon^{2} p_{4}^{2}\left(p_{4}^{2}-m_{B}^{2}\right)}{2 m_{B}^{2}+p_{4}^{2}-s} \mathcal{T}_{3}-\frac{2 \epsilon^{2} p_{4}^{2} s}{2 m_{B}^{2}+p_{4}^{2}-s} \mathcal{T}_{4}+\frac{2 \epsilon^{2} s\left(4 m_{B}^{2}+3 p_{4}^{2}-2 s\right)}{2 m_{B}^{2}+p_{4}^{2}-s} \mathcal{T}_{7} \\
& -\frac{p_{4}^{2}(\epsilon-1) \epsilon^{2}}{p_{4}^{2}-2 s} \mathcal{T}_{8}+\frac{\epsilon^{2} p_{4}^{2}\left(m_{B}^{2}-s\right)}{2\left(p_{4}^{2}-2 s\right)} \mathcal{T}_{9}+\frac{\epsilon^{2} p_{4}^{2}\left(m_{B}^{2}-s\right)}{p_{4}^{2}-2 s} \mathcal{T}_{10}+2 \epsilon^{2} m_{B}^{2} p_{4}^{2} \mathcal{T}_{13} \\
& +2 \epsilon^{2} p_{4}^{2} s \mathcal{T}_{14}+\frac{\epsilon^{3} p_{4}^{2}\left(7 s-5 p_{4}^{2}\right)}{p_{4}^{2}-2 s} \mathcal{T}_{15}+\frac{2 \epsilon^{2} p_{4}^{2}\left(m_{B}^{2} p_{4}^{2}+s\left(s-p_{4}^{2}\right)\right)}{p_{4}^{2}-2 s} \mathcal{T}_{16} \\
& -\frac{4 \epsilon^{3} p_{4}^{2}\left(m_{B}^{2}-p_{4}^{2}+s\right)}{2 m_{B}^{2}+p_{4}^{2}-s} \mathcal{T}_{21}+\frac{2 m_{B}^{2} p_{4}^{2} \epsilon^{2}\left(m_{B}^{2}-p_{4}^{2}+s\right)}{2 m_{B}^{2}+p_{4}^{2}-s} \mathcal{T}_{22} \\
& f_{34}=\epsilon^{3} \sqrt{p_{4}^{2}} \sqrt{4 m_{B}^{2}-p_{4}^{2}}\left(s-p_{4}^{2}\right) \mathcal{T}_{34},
\end{aligned}
$$




$$
\begin{aligned}
& f_{35}=\epsilon^{4}(s+t) \mathcal{T}_{35}, \\
& f_{36}=\epsilon^{3}\left(-\left(m_{B}^{2}(s+t)-s t\right)\right) \mathcal{T}_{36}+2 \epsilon^{4}(s+t) \mathcal{T}_{35}, \\
& f_{37}=\epsilon^{3} t\left(s-m_{B}^{2}\right) \mathcal{T}_{37}+\epsilon^{3}\left(s-m_{B}^{2}\right) t \mathcal{T}_{38}, \\
& f_{38}=\epsilon^{3} s t \mathcal{T}_{38} \text {, } \\
& f_{39}=\epsilon^{3} s\left(t-m_{B}^{2}\right) \mathcal{T}_{39}+\epsilon^{3} s\left(t-m_{B}^{2}\right) \mathcal{T}_{40}, \\
& f_{40}=\epsilon^{3} s t \mathcal{T}_{40} \text {, } \\
& f_{41}=\epsilon^{3} \sqrt{p_{4}^{2}} \sqrt{4 m_{B}^{2}-p_{4}^{2}} s t \mathcal{T}_{41}, \\
& f_{42}=\epsilon^{4} t\left(s-p_{4}^{2}\right) \mathcal{T}_{42}, \\
& f_{43}=\epsilon^{4} t \mathcal{T}_{43}+\epsilon^{4} p_{4}^{2} t \mathcal{T}_{42}-\epsilon^{4} t \mathcal{T}_{35}, \\
& f_{44}=-\frac{\epsilon^{3} \sqrt{p_{4}^{2}} \sqrt{4 m_{B}^{2}-p_{4}^{2}} t\left(m_{B}^{2} p_{4}^{2}+s\left(s-p_{4}^{2}\right)\right)}{p_{4}^{2}-2 s} \mathcal{T}_{44}+\frac{\epsilon^{4} \sqrt{p_{4}^{2}} \sqrt{4 m_{B}^{2}-p_{4}^{2}} t}{p_{4}^{2}-2 s} \mathcal{T}_{43} \\
& +\frac{\epsilon^{4} \sqrt{p_{4}^{2}} \sqrt{4 m_{B}^{2}-p_{4}^{2}}\left(2 p_{4}^{2}-s\right) t}{p_{4}^{2}-2 s} \mathcal{T}_{42}+\frac{\epsilon^{2}\left(m_{B}^{2}-3 p_{4}^{2}\right) \sqrt{p_{4}^{2}} \sqrt{4 m_{B}^{2}-p_{4}^{2}} t}{3\left(p_{4}^{2}-2 s\right)\left(2 m_{B}^{2}+p_{4}^{2}-t\right)} \mathcal{T}_{2} \\
& -\frac{\epsilon^{2}\left(m_{B}^{2}-p_{4}^{2}\right) \sqrt{p_{4}^{2}} \sqrt{4 m_{B}^{2}-p_{4}^{2}}\left(10 m_{B}^{2}-3\left(3 p_{4}^{2}+t\right)\right)}{12\left(p_{4}^{2}-2 s\right)\left(2 m_{B}^{2}+p_{4}^{2}-t\right)} \mathcal{T}_{3} \\
& +\frac{\epsilon^{2} \sqrt{p_{4}^{2}} \sqrt{4 m_{B}^{2}-p_{4}^{2}}\left(-10 m_{B}^{4}+m_{B}^{2}\left(p_{4}^{2}+3 t\right)+3 p_{4}^{2}\left(p_{4}^{2}+t\right)\right)}{6\left(p_{4}^{2}-2 s\right)\left(2 m_{B}^{2}+p_{4}^{2}-t\right)} \mathcal{T}_{4} \\
& +\frac{\epsilon^{2} \sqrt{p_{4}^{2}} \sqrt{4 m_{B}^{2}-p_{4}^{2}}\left(t-m_{B}^{2}\right)\left(3 p_{4}^{2}-2 t\right)}{8\left(p_{4}^{2}-2 s\right)\left(p_{4}^{2}-2 t\right)} \mathcal{T}_{5} \\
& +\frac{\epsilon^{2} \sqrt{p_{4}^{2}} \sqrt{4 m_{B}^{2}-p_{4}^{2}}\left(2 t\left(m_{B}^{2}+p_{4}^{2}\right)-3 m_{B}^{2} p_{4}^{2}\right)}{4\left(p_{4}^{2}-2 s\right)\left(p_{4}^{2}-2 t\right)} \mathcal{T}_{6} \\
& +\frac{(\epsilon-1) \epsilon^{2} \sqrt{p_{4}^{2}} \sqrt{4 m_{B}^{2}-p_{4}^{2}}\left(11 p_{4}^{2}-16 t\right)}{6\left(p_{4}^{2}-2 s\right)\left(p_{4}^{2}-2 t\right)} \mathcal{T}_{8}+\frac{\epsilon^{2} \sqrt{p_{4}^{2}} \sqrt{4 m_{B}^{2}-p_{4}^{2}}\left(s-m_{B}^{2}\right)}{8\left(p_{4}^{2}-2 s\right)} \mathcal{T}_{9} \\
& -\frac{\epsilon^{2} m_{B}^{2} \sqrt{p_{4}^{2}} \sqrt{4 m_{B}^{2}-p_{4}^{2}}}{4 p_{4}^{2}-8 s} \mathcal{T}_{10}-\frac{\epsilon^{2} \sqrt{\left(p_{4}^{2}\right)^{3}} \sqrt{4 m_{B}^{2}-p_{4}^{2}} t}{p_{4}^{2}-2 s} \mathcal{T}_{12} \\
& -\frac{\epsilon^{2} m_{B}^{2} \sqrt{\left(p_{4}^{2}\right)^{3}} \sqrt{4 m_{B}^{2}-p_{4}^{2}}}{p_{4}^{2}-2 s} \mathcal{T}_{13}+\frac{\epsilon^{3} \sqrt{p_{4}^{2}} \sqrt{4 m_{B}^{2}-p_{4}^{2}}\left(s-p_{4}^{2}\right)}{2 p_{4}^{2}-4 s} \mathcal{T}_{15} \\
& +\frac{\epsilon^{3} \sqrt{p_{4}^{2}} \sqrt{4 m_{B}^{2}-p_{4}^{2}}\left(2 p_{4}^{4}-2 p_{4}^{2} t-t^{2}\right)}{\left(p_{4}^{2}-2 s\right)\left(p_{4}^{2}-2 t\right)} \mathcal{T}_{17} \\
& -\frac{\epsilon^{2} \sqrt{\left(p_{4}^{2}\right)^{3}} \sqrt{4 m_{B}^{2}-p_{4}^{2}}\left(m_{B}^{2} p_{4}^{2}+t\left(t-p_{4}^{2}\right)\right)}{\left(p_{4}^{2}-2 s\right)\left(p_{4}^{2}-2 t\right)} \mathcal{T}_{18}
\end{aligned}
$$




$$
\begin{aligned}
& +\frac{\epsilon^{3} \sqrt{p_{4}^{2}} \sqrt{4 m_{B}^{2}-p_{4}^{2}}\left(m_{B}^{2}\left(3 p_{4}^{2}-t\right)+2 p_{4}^{2}\left(t-p_{4}^{2}\right)\right)}{\left(p_{4}^{2}-2 s\right)\left(2 m_{B}^{2}+p_{4}^{2}-t\right)} \mathcal{T}_{19} \\
& -\frac{\epsilon^{2} m_{B}^{2} \sqrt{p_{4}^{2}} \sqrt{4 m_{B}^{2}-p_{4}^{2}}\left(7 p_{4}^{2}-t\right)\left(m_{B}^{2}-p_{4}^{2}+t\right)}{6\left(p_{4}^{2}-2 s\right)\left(2 m_{B}^{2}+p_{4}^{2}-t\right)} \mathcal{T}_{20} \\
& +\frac{\epsilon^{4} \sqrt{p_{4}^{2}} \sqrt{4 m_{B}^{2}-p_{4}^{2}}\left(p_{4}^{2}-s\right)}{p_{4}^{2}-2 s} \mathcal{T}_{26} \\
& -\frac{\epsilon^{4} \sqrt{p_{4}^{2}} \sqrt{4 m_{B}^{2}-p_{4}^{2}}\left(p_{4}^{2}+t\right)}{p_{4}^{2}-2 s} \mathcal{T}_{27}-\frac{\epsilon^{2} m_{B}^{2} \sqrt{\left(p_{4}^{2}\right)^{3}} \sqrt{4 m_{B}^{2}-p_{4}^{2}} t}{p_{4}^{2}-2 s} \mathcal{T}_{29} \\
& -\frac{\epsilon^{3} \sqrt{\left(p_{4}^{2}\right)^{3}} \sqrt{4 m_{B}^{2}-p_{4}^{2}}\left(p_{4}^{2}+t\right)}{2\left(p_{4}^{2}-2 s\right)} \mathcal{T}_{30}+\frac{\epsilon^{4} \sqrt{p_{4}^{2}} \sqrt{4 m_{B}^{2}-p_{4}^{2}}(3 s+t)}{2\left(p_{4}^{2}-2 s\right)} \mathcal{T}_{35} \\
& +\frac{\epsilon^{3} \sqrt{p_{4}^{2}} \sqrt{4 m_{B}^{2}-p_{4}^{2}}\left(m_{B}^{2}(s+t)-s t\right)}{4\left(p_{4}^{2}-2 s\right)} \mathcal{T}_{36} \\
& +\frac{\epsilon^{3} \sqrt{p_{4}^{2}} \sqrt{4 m_{B}^{2}-p_{4}^{2}} t\left(s-m_{B}^{2}\right)}{6\left(p_{4}^{2}-2 s\right)} \mathcal{T}_{37}-\frac{\epsilon^{3} \sqrt{p_{4}^{2}} \sqrt{4 m_{B}^{2}-p_{4}^{2}}\left(m_{B}^{2}-3 s\right) t}{6\left(p_{4}^{2}-2 s\right)} \mathcal{T}_{38}, \\
& f_{45}=\epsilon^{4} s\left(t-p_{4}^{2}\right) \mathcal{T}_{45}, \\
& f_{46}=\epsilon^{4} s \mathcal{T}_{46}+\epsilon^{4} p_{4}^{2} s \mathcal{T}_{45}-\epsilon^{4} s \mathcal{T}_{35}, \\
& f_{47}=-\frac{\epsilon^{3} \sqrt{p_{4}^{2}} \sqrt{4 m_{B}^{2}-p_{4}^{2}} s\left(m_{B}^{2} p_{4}^{2}+t\left(t-p_{4}^{2}\right)\right)}{p_{4}^{2}-2 t} \mathcal{T}_{47}+\frac{\epsilon^{4} \sqrt{p_{4}^{2}} \sqrt{4 m_{B}^{2}-p_{4}^{2}} s}{p_{4}^{2}-2 t} \mathcal{T}_{46} \\
& +\frac{\epsilon^{4} \sqrt{p_{4}^{2}} \sqrt{4 m_{B}^{2}-p_{4}^{2}}\left(2 p_{4}^{2}-t\right) s}{p_{4}^{2}-2 t} \mathcal{T}_{45} \\
& -\frac{\epsilon^{2} \sqrt{p_{4}^{2}} \sqrt{4 m_{B}^{2}-p_{4}^{2}}\left(m_{B}^{2}-p_{4}^{2}\right)\left(10 m_{B}^{2}-3\left(3 p_{4}^{2}+s\right)\right)}{12\left(p_{4}^{2}-2 t\right)\left(2 m_{B}^{2}+p_{4}^{2}-s\right)} \mathcal{T}_{3} \\
& +\frac{\epsilon^{2} \sqrt{p_{4}^{2}} \sqrt{4 m_{B}^{2}-p_{4}^{2}}\left(-10 m_{B}^{4}+m_{B}^{2}\left(p_{4}^{2}+3 s\right)+3 p_{4}^{2}\left(p_{4}^{2}+s\right)\right)}{6\left(p_{4}^{2}-2 t\right)\left(2 m_{B}^{2}+p_{4}^{2}-s\right)} \mathcal{T}_{4} \\
& +\frac{\epsilon^{2} \sqrt{p_{4}^{2}} \sqrt{4 m_{B}^{2}-p_{4}^{2}}\left(t-m_{B}^{2}\right)}{8\left(p_{4}^{2}-2 t\right)} \mathcal{T}_{5}-\frac{\epsilon^{2} m_{B}^{2} \sqrt{p_{4}^{2}} \sqrt{4 m_{B}^{2}-p_{4}^{2}}}{4 p_{4}^{2}-8 t} \mathcal{T}_{6} \\
& +\frac{\epsilon^{2} \sqrt{p_{4}^{2}} \sqrt{4 m_{B}^{2}-p_{4}^{2}} s\left(m_{B}^{2}-3 p_{4}^{2}\right)}{3\left(p_{4}^{2}-2 t\right)\left(2 m_{B}^{2}+p_{4}^{2}-s\right)} \mathcal{T}_{7}+\frac{(\epsilon-1) \epsilon^{2} \sqrt{p_{4}^{2}} \sqrt{4 m_{B}^{2}-p_{4}^{2}}\left(11 p_{4}^{2}-16 s\right)}{6\left(p_{4}^{2}-2 s\right)\left(p_{4}^{2}-2 t\right)} \mathcal{T}_{8} \\
& +\frac{\epsilon^{2} \sqrt{p_{4}^{2}} \sqrt{4 m_{B}^{2}-p_{4}^{2}}\left(s-m_{B}^{2}\right)\left(3 p_{4}^{2}-2 s\right)}{8\left(p_{4}^{2}-2 s\right)\left(p_{4}^{2}-2 t\right)} \mathcal{T}_{9} \\
& +\frac{\epsilon^{2} \sqrt{p_{4}^{2}} \sqrt{4 m_{B}^{2}-p_{4}^{2}}\left(2 s\left(m_{B}^{2}+p_{4}^{2}\right)-3 m_{B}^{2} p_{4}^{2}\right)}{4\left(p_{4}^{2}-2 s\right)\left(p_{4}^{2}-2 t\right)} \mathcal{T}_{10}-\frac{\epsilon^{2} m_{B}^{2}{\sqrt{p_{4}^{2}}}^{3} \sqrt{4 m_{B}^{2}-p_{4}^{2}}}{p_{4}^{2}-2 t} \mathcal{T}_{13}
\end{aligned}
$$




$$
\begin{aligned}
& -\frac{\epsilon^{2}{\sqrt{p_{4}^{2}}}^{3} \sqrt{4 m_{B}^{2}-p_{4}^{2}} s}{p_{4}^{2}-2 t} \mathcal{T}_{14}+\frac{\epsilon^{3} \sqrt{p_{4}^{2}} \sqrt{4 m_{B}^{2}-p_{4}^{2}}\left(2 p_{4}^{4}-2 p_{4}^{2} s-s^{2}\right)}{\left(p_{4}^{2}-2 s\right)\left(p_{4}^{2}-2 t\right)} \mathcal{T}_{15} \\
& -\frac{\epsilon^{2} \sqrt{p_{4}^{2}} \sqrt{4 m_{B}^{2}-p_{4}^{2}}\left(m_{B}^{2} p_{4}^{2}+s\left(s-p_{4}^{2}\right)\right)}{\left(p_{4}^{2}-2 s\right)\left(p_{4}^{2}-2 t\right)} \mathcal{T}_{16}+\frac{\epsilon^{3} \sqrt{p_{4}^{2}} \sqrt{4 m_{B}^{2}-p_{4}^{2}}\left(t-p_{4}^{2}\right)}{2 p_{4}^{2}-4 t} \mathcal{T}_{17} \\
& +\frac{\epsilon^{3} \sqrt{p_{4}^{2}} \sqrt{4 m_{B}^{2}-p_{4}^{2}}\left(m_{B}^{2}\left(3 p_{4}^{2}-s\right)+2 p_{4}^{2}\left(s-p_{4}^{2}\right)\right)}{\left(p_{4}^{2}-2 t\right)\left(2 m_{B}^{2}+p_{4}^{2}-s\right)} \mathcal{T}_{21} \\
& -\frac{\epsilon^{2} m_{B}^{2} \sqrt{p_{4}^{2}} \sqrt{4 m_{B}^{2}-p_{4}^{2}}\left(7 p_{4}^{2}-s\right)\left(m_{B}^{2}-p_{4}^{2}+s\right)}{6\left(p_{4}^{2}-2 t\right)\left(2 m_{B}^{2}+p_{4}^{2}-s\right)} \mathcal{T}_{22} \\
& +\frac{\epsilon^{4} \sqrt{p_{4}^{2}} \sqrt{4 m_{B}^{2}-p_{4}^{2}}\left(p_{4}^{2}-t\right)}{p_{4}^{2}-2 t} \mathcal{T}_{24}-\frac{\epsilon^{4} \sqrt{p_{4}^{2}} \sqrt{4 m_{B}^{2}-p_{4}^{2}}\left(p_{4}^{2}+s\right)}{p_{4}^{2}-2 t} \mathcal{T}_{31} \\
& -\frac{\epsilon^{2} m_{B}^{2} s{\sqrt{p_{4}^{2}}}^{3} \sqrt{4 m_{B}^{2}-p_{4}^{2}}}{p_{4}^{2}-2 t} \mathcal{T}_{33}-\frac{\epsilon^{3}{\sqrt{p_{4}^{2}}}^{3} \sqrt{4 m_{B}^{2}-p_{4}^{2}}\left(p_{4}^{2}+s\right)}{2\left(p_{4}^{2}-2 t\right)} \mathcal{T}_{34} \\
& +\frac{\epsilon^{4} \sqrt{p_{4}^{2}} \sqrt{4 m_{B}^{2}-p_{4}^{2}}(s+3 t)}{2\left(p_{4}^{2}-2 t\right)} \mathcal{T}_{35}+\frac{\epsilon^{3} \sqrt{p_{4}^{2}} \sqrt{4 m_{B}^{2}-p_{4}^{2}}\left(m_{B}^{2}(s+t)-s t\right)}{4\left(p_{4}^{2}-2 t\right)} \mathcal{T}_{36} \\
& +\frac{\epsilon^{3} \sqrt{p_{4}^{2}} \sqrt{4 m_{B}^{2}-p_{4}^{2}} s\left(t-m_{B}^{2}\right)}{6\left(p_{4}^{2}-2 t\right)} \mathcal{T}_{39}-\frac{\epsilon^{3} \sqrt{p_{4}^{2}} \sqrt{4 m_{B}^{2}-p_{4}^{2}} s\left(m_{B}^{2}-3 t\right)}{6\left(p_{4}^{2}-2 t\right)} \mathcal{T}_{40}, \\
& f_{48}=\epsilon^{4} p_{4}^{2} s t \mathcal{T}_{48}+\frac{\epsilon^{2}\left(m_{B}^{2}-3 p_{4}^{2}\right) \sqrt{p_{4}^{2}} \sqrt{4 m_{B}^{2}-p_{4}^{2}} t}{3\left(p_{4}^{2}-2 s\right)\left(2 m_{B}^{2}+p_{4}^{2}-t\right)} \mathcal{T}_{2}+A\left(s, t, p_{4}^{2}\right) \mathcal{T}_{3}+B\left(s, t, p_{4}^{2}\right) \mathcal{T}_{4} \\
& +\frac{\epsilon^{2} \sqrt{p_{4}^{2}} \sqrt{4 m_{B}^{2}-p_{4}^{2}}\left(m_{B}^{2}-t\right)\left(-2 p_{4}^{2}+s+t\right)}{4\left(p_{4}^{2}-2 s\right)\left(p_{4}^{2}-2 t\right)} \mathcal{T}_{5} \\
& +\frac{\epsilon^{2} \sqrt{p_{4}^{2}} \sqrt{4 m_{B}^{2}-p_{4}^{2}}\left(m_{B}^{2}\left(-2 p_{4}^{2}+s+t\right)+p_{4}^{2} t\right)}{2\left(p_{4}^{2}-2 s\right)\left(p_{4}^{2}-2 t\right)} \mathcal{T}_{6} \\
& +\frac{(\epsilon-1) \epsilon^{2} \sqrt{p_{4}^{2}} \sqrt{4 m_{B}^{2}-p_{4}^{2}}\left(11 p_{4}^{2}-8(s+t)\right)}{3\left(p_{4}^{2}-2 s\right)\left(p_{4}^{2}-2 t\right)} \mathcal{T}_{8} \\
& +\frac{\epsilon^{2} \sqrt{p_{4}^{2}} \sqrt{4 m_{B}^{2}-p_{4}^{2}}\left(m_{B}^{2}-s\right)\left(-2 p_{4}^{2}+s+t\right)}{4\left(p_{4}^{2}-2 s\right)\left(p_{4}^{2}-2 t\right)} \mathcal{T}_{9} \\
& +\frac{\epsilon^{2} \sqrt{p_{4}^{2}} \sqrt{4 m_{B}^{2}-p_{4}^{2}}\left(m_{B}^{2}\left(-2 p_{4}^{2}+s+t\right)+p_{4}^{2} s\right)}{2\left(p_{4}^{2}-2 s\right)\left(p_{4}^{2}-2 t\right)} \mathcal{T}_{10}-\frac{\epsilon^{2}{\sqrt{p_{4}^{2}}}^{3} \sqrt{4 m_{B}^{2}-p_{4}^{2}} t}{p_{4}^{2}-2 s} \mathcal{T}_{12} \\
& +\frac{2 \epsilon^{2} m_{B}^{2}{ }^{3 / 2} \sqrt{4 m_{B}^{2}-p_{4}^{2}}\left(-p_{4}^{2}+s+t\right)}{\left(p_{4}^{2}-2 s\right)\left(p_{4}^{2}-2 t\right)} \mathcal{T}_{13}-\frac{\epsilon^{2} \sqrt{p_{4}^{2}} \sqrt{4 m_{B}^{2}-p_{4}^{2}} s}{p_{4}^{2}-2 t} \mathcal{T}_{14} \\
& +\frac{\epsilon^{3} \sqrt{p_{4}^{2}} \sqrt{4 m_{B}^{2}-p_{4}^{2}}\left(3 p_{4}^{4}+p_{4}^{2}(2 t-3 s)-2 s(s+t)\right)}{2\left(p_{4}^{2}-2 s\right)\left(p_{4}^{2}-2 t\right)} \mathcal{T}_{15}
\end{aligned}
$$




$$
\begin{aligned}
& -\frac{\epsilon^{2}{\sqrt{p_{4}^{2}}}^{3} \sqrt{4 m_{B}^{2}-p_{4}^{2}}\left(m_{B}^{2} p_{4}^{2}+s\left(s-p_{4}^{2}\right)\right)}{\left(p_{4}^{2}-2 s\right)\left(p_{4}^{2}-2 t\right)} \mathcal{T}_{16} \\
& +\frac{\epsilon^{3} \sqrt{p_{4}^{2}} \sqrt{4 m_{B}^{2}-p_{4}^{2}}\left(3 p_{4}^{4}+p_{4}^{2}(2 s-3 t)-2 t(s+t)\right)}{2\left(p_{4}^{2}-2 s\right)\left(p_{4}^{2}-2 t\right)} \mathcal{T}_{17} \\
& -\frac{\epsilon^{2}{\sqrt{p_{4}^{2}}}^{3} \sqrt{4 m_{B}^{2}-p_{4}^{2}}\left(m_{B}^{2} p_{4}^{2}+t\left(t-p_{4}^{2}\right)\right)}{\left(p_{4}^{2}-2 s\right)\left(p_{4}^{2}-2 t\right)} \mathcal{T}_{18} \\
& +\frac{\epsilon^{3} \sqrt{p_{4}^{2}} \sqrt{4 m_{B}^{2}-p_{4}^{2}}\left(m_{B}^{2}\left(3 p_{4}^{2}-t\right)+2 p_{4}^{2}\left(t-p_{4}^{2}\right)\right)}{\left(p_{4}^{2}-2 s\right)\left(2 m_{B}^{2}+p_{4}^{2}-t\right)} \mathcal{T}_{19} \\
& -\frac{\epsilon^{2} m_{B}^{2} \sqrt{p_{4}^{2}} \sqrt{4 m_{B}^{2}-p_{4}^{2}}\left(7 p_{4}^{2}-t\right)\left(m_{B}^{2}-p_{4}^{2}+t\right)}{6\left(p_{4}^{2}-2 s\right)\left(2 m_{B}^{2}+p_{4}^{2}-t\right)} \mathcal{T}_{20} \\
& +\frac{\epsilon^{3} \sqrt{p_{4}^{2}} \sqrt{4 m_{B}^{2}-p_{4}^{2}}\left(m_{B}^{2}\left(3 p_{4}^{2}-s\right)+2 p_{4}^{2}\left(s-p_{4}^{2}\right)\right)}{\left(p_{4}^{2}-2 t\right)\left(2 m_{B}^{2}+p_{4}^{2}-s\right)} \mathcal{T}_{21} \\
& -\frac{\epsilon^{2} m_{B}^{2} \sqrt{p_{4}^{2}} \sqrt{4 m_{B}^{2}-p_{4}^{2}}\left(7 p_{4}^{2}-s\right)\left(m_{B}^{2}-p_{4}^{2}+s\right)}{6\left(p_{4}^{2}-2 t\right)\left(2 m_{B}^{2}+p_{4}^{2}-s\right)} \mathcal{T}_{22} \\
& +\frac{\epsilon^{4} \sqrt{p_{4}^{2}} \sqrt{4 m_{B}^{2}-p_{4}^{2}}\left(p_{4}^{2}-t\right)}{p_{4}^{2}-2 t} \mathcal{T}_{24}+\frac{\epsilon^{4} \sqrt{p_{4}^{2}} \sqrt{4 m_{B}^{2}-p_{4}^{2}}\left(p_{4}^{2}-s\right)}{p_{4}^{2}-2 s} \mathcal{T}_{26} \\
& -\frac{\epsilon^{4} \sqrt{p_{4}^{2}} \sqrt{4 m_{B}^{2}-p_{4}^{2}}\left(p_{4}^{2}+t\right)}{p_{4}^{2}-2 s} \mathcal{T}_{27}+\epsilon^{3} m_{B}^{2}\left(t-p_{4}^{2}\right) \mathcal{T}_{28} \\
& -\frac{\epsilon^{2} m_{B}^{2}{\sqrt{p_{4}^{2}}}^{3} \sqrt{4 m_{B}^{2}-p_{4}^{2}} t}{p_{4}^{2}-2 s} \mathcal{T}_{29}-\frac{\epsilon^{3}{\sqrt{p_{4}^{2}}}^{3} \sqrt{4 m_{B}^{2}-p_{4}^{2}}\left(p_{4}^{2}+t\right)}{2\left(p_{4}^{2}-2 s\right)} \mathcal{T}_{30} \\
& -\frac{\epsilon^{4} \sqrt{p_{4}^{2}} \sqrt{4 m_{B}^{2}-p_{4}^{2}}\left(p_{4}^{2}+s\right)}{p_{4}^{2}-2 t} \mathcal{T}_{31}+\epsilon^{3} s\left(s-p_{4}^{2}\right) \mathcal{T}_{32} \\
& -\frac{\epsilon^{2} m_{B}^{2}{\sqrt{p_{4}^{2}}}^{3} \sqrt{4 m_{B}^{2}-p_{4}^{2}} s}{p_{4}^{2}-2 t} \mathcal{T}_{33}-\frac{\epsilon^{3}{\sqrt{p_{4}^{2}}}^{3} \sqrt{4 m_{B}^{2}-p_{4}^{2}}\left(p_{4}^{2}+s\right)}{2\left(p_{4}^{2}-2 t\right)} \mathcal{T}_{34} \\
& +\frac{\epsilon^{4} \sqrt{p_{4}^{2}} \sqrt{4 m_{B}^{2}-p_{4}^{2}}\left(2 p_{4}^{2}(s+t)-s^{2}-6 s t-t^{2}\right)}{\left(p_{4}^{2}-2 s\right)\left(p_{4}^{2}-2 t\right)} \mathcal{T}_{35} \\
& +\frac{\epsilon^{3} \sqrt{p_{4}^{2}} \sqrt{4 m_{B}^{2}-p_{4}^{2}}\left(m_{B}^{2}(s+t)-s t\right)\left(p_{4}^{2}-s-t\right)}{2\left(p_{4}^{2}-2 s\right)\left(p_{4}^{2}-2 t\right)} \mathcal{T}_{36} \\
& +\frac{\epsilon^{3} \sqrt{p_{4}^{2}} \sqrt{4 m_{B}^{2}-p_{4}^{2}} t\left(s-m_{B}^{2}\right)}{6\left(p_{4}^{2}-2 s\right)} \mathcal{T}_{37}-\frac{\epsilon^{3} \sqrt{p_{4}^{2}} \sqrt{4 m_{B}^{2}-p_{4}^{2}}\left(m_{B}^{2}-3 s\right) t}{6\left(p_{4}^{2}-2 s\right)} \mathcal{T}_{38} \\
& +\frac{\epsilon^{3} \sqrt{p_{4}^{2}} \sqrt{4 m_{B}^{2}-p_{4}^{2}} s\left(t-m_{B}^{2}\right)}{6\left(p_{4}^{2}-2 t\right)} \mathcal{T}_{39}-\frac{\epsilon^{3} \sqrt{p_{4}^{2}} \sqrt{4 m_{B}^{2}-p_{4}^{2}} s\left(m_{B}^{2}-3 t\right)}{6\left(p_{4}^{2}-2 t\right)} \mathcal{T}_{40}
\end{aligned}
$$




$$
\begin{aligned}
& +\frac{\epsilon^{4} \sqrt{p_{4}^{2}} t\left(-s \sqrt{4 m_{B}^{2}-p_{4}^{2}}+2 p_{4}^{2} \sqrt{4 m_{B}^{2}-p_{4}^{2}}+\sqrt{p_{4}^{2}}\left(p_{4}^{2}-2 s\right)\right)}{p_{4}^{2}-2 s} \mathcal{T}_{42} \\
& +\frac{\epsilon^{4} \sqrt{p_{4}^{2}} \sqrt{4 m_{B}^{2}-p_{4}^{2}} t}{p_{4}^{2}-2 s} \mathcal{T}_{43}-\frac{\epsilon^{3} \sqrt{p_{4}^{2}} \sqrt{4 m_{B}^{2}-p_{4}^{2}} t\left(m_{B}^{2} p_{4}^{2}+s\left(s-p_{4}^{2}\right)\right)}{p_{4}^{2}-2 s} \mathcal{T}_{44} \\
& +\frac{\epsilon^{4} s \sqrt{p_{4}^{2}}\left(-t \sqrt{4 m_{B}^{2}-p_{4}^{2}}+2 p_{4}^{2} \sqrt{4 m_{B}^{2}-p_{4}^{2}}+\sqrt{p_{4}^{2}}\left(p_{4}^{2}-2 t\right)\right)}{p_{4}^{2}-2 t} \mathcal{T}_{45} \\
& +\frac{\epsilon^{4} s \sqrt{p_{4}^{2}} \sqrt{4 m_{B}^{2}-p_{4}^{2}}}{p_{4}^{2}-2 t} \mathcal{T}_{46}-\frac{\epsilon^{3} s \sqrt{p_{4}^{2}} \sqrt{4 m_{B}^{2}-p_{4}^{2}}\left(m_{B}^{2} p_{4}^{2}+t\left(t-p_{4}^{2}\right)\right)}{p_{4}^{2}-2 t} \mathcal{T}_{47},
\end{aligned}
$$

where

$$
\begin{aligned}
A\left(s, t, p_{4}^{2}\right)= & -\frac{\left(m_{B}^{2}-p_{4}^{2}\right) \sqrt{p_{4}^{2}} \sqrt{4 m_{B}^{2}-p_{4}^{2}}\left(20 m_{B}^{4}\left(p_{4}^{2}-s-t\right)+m_{B}^{2}\left(2(3 s+t)(s+3 t)-8 p_{4}^{4}\right)\right)}{6\left(p_{4}^{2}-2 s\right)\left(p_{4}^{2}-2 t\right)\left(2 m_{B}^{2}+p_{4}^{2}-s\right)\left(2 m_{B}^{2}+p_{4}^{2}-t\right)} \\
& +\frac{\left(m_{B}^{2}-p_{4}^{2}\right) \sqrt{p_{4}^{2}} \sqrt{4 m_{B}^{2}-p_{4}^{2}}\left(3 p_{4}^{6}-4 p_{4}^{4}(s+t)-p_{4}^{2}\left(s^{2}-5 s t+t^{2}\right)+s t(s+t)\right)}{2\left(p_{4}^{2}-2 s\right)\left(p_{4}^{2}-2 t\right)\left(2 m_{B}^{2}+p_{4}^{2}-s\right)\left(2 m_{B}^{2}+p_{4}^{2}-t\right)} \\
B\left(s, t, p_{4}^{2}\right)=- & \frac{\sqrt{p_{4}^{2}} \sqrt{4 m_{B}^{2}-p_{4}^{2}}\left(2 m_{B}^{2}+p_{4}^{2}\right) s\left(-10 m_{B}^{4}-3 m_{B}^{2} p_{4}^{2}+3 p_{4}^{4}\right)}{3\left(p_{4}^{2}-2 s\right)\left(p_{4}^{2}-2 t\right)\left(2 m_{B}^{2}+p_{4}^{2}-s\right)\left(2 m_{B}^{2}+p_{4}^{2}-t\right)} \\
& -\frac{\sqrt{p_{4}^{2}} \sqrt{4 m_{B}^{2}-p_{4}^{2}}\left(2 m_{B}^{2}+p_{4}^{2}\right)\left(3 s^{2}\left(m_{B}^{2}+p_{4}^{2}\right)-p_{4}^{2}\left(2 m_{B}^{2}+p_{4}^{2}\right)\left(3 p_{4}^{2}-5 m_{B}^{2}\right)\right)}{3\left(p_{4}^{2}-2 s\right)\left(p_{4}^{2}-2 t\right)\left(2 m_{B}^{2}+p_{4}^{2}-s\right)\left(2 m_{B}^{2}+p_{4}^{2}-t\right)} \\
& -\frac{t^{2} \sqrt{p_{4}^{2}} \sqrt{4 m_{B}^{2}-p_{4}^{2}}\left(m_{B}^{2}+p_{4}^{2}\right)}{\left(p_{4}^{2}-2 s\right)\left(p_{4}^{2}-2 t\right)\left(2 m_{B}^{2}+p_{4}^{2}-t\right)} \\
& +\frac{t \sqrt{p_{4}^{2}} \sqrt{4 m_{B}^{2}-p_{4}^{2}}\left(2 m_{B}^{2}+p_{4}^{2}\right)\left(10 m_{B}^{4}+3 m_{B}^{2} p_{4}^{2}-3 p_{4}^{4}\right)}{3\left(p_{4}^{2}-2 s\right)\left(p_{4}^{2}-2 t\right)\left(2 m_{B}^{2}+p_{4}^{2}-s\right)\left(2 m_{B}^{2}+p_{4}^{2}-t\right)} \\
& -\frac{s t \sqrt{p_{4}^{2}} \sqrt{4 m_{B}^{2}-p_{4}^{2}}\left(20 m_{B}^{4}+m_{B}^{2}\left(p_{4}^{2}-3 s\right)-3 p_{4}^{2}\left(p_{4}^{2}+s\right)\right)}{3\left(p_{4}^{2}-2 s\right)\left(p_{4}^{2}-2 t\right)\left(2 m_{B}^{2}+p_{4}^{2}-s\right)\left(2 m_{B}^{2}+p_{4}^{2}-t\right)} .
\end{aligned}
$$

Open Access. This article is distributed under the terms of the Creative Commons Attribution License (CC-BY 4.0), which permits any use, distribution and reproduction in any medium, provided the original author(s) and source are credited.

\section{References}

[1] ATLAS collaboration, Observation of a new particle in the search for the Standard Model Higgs boson with the ATLAS detector at the LHC, Phys. Lett. B 716 (2012) 1 [arXiv: 1207.7214] [INSPIRE].

[2] CMS collaboration, Observation of a new boson at a mass of $125 \mathrm{GeV}$ with the CMS experiment at the LHC, Phys. Lett. B 716 (2012) 30 [arXiv:1207.7235] [INSPIRE]. 
[3] LHC Higgs Cross Section Working Group collaboration, S. Dittmaier et al., Handbook of LHC Higgs Cross Sections: 1. Inclusive Observables, arXiv:1101.0593 [INSPIRE].

[4] S. Dittmaier et al., Handbook of LHC Higgs Cross Sections: 2. Differential Distributions, arXiv:1201.3084 [INSPIRE].

[5] LhC Higgs Cross Section Working Group collaboration, J.R. Andersen et al., Handbook of LHC Higgs Cross Sections: 3. Higgs Properties, arXiv:1307.1347 [INSPIRE].

[6] LHC Higgs Cross Section Working Group collaboration, D. de Florian et al., Handbook of LHC Higgs Cross Sections: 4. Deciphering the Nature of the Higgs Sector, arXiv: 1610.07922 [INSPIRE].

[7] D. Graudenz, M. Spira and P.M. Zerwas, QCD corrections to Higgs boson production at proton proton colliders, Phys. Rev. Lett. 70 (1993) 1372 [INSPIRE].

[8] M. Spira, A. Djouadi, D. Graudenz and P.M. Zerwas, Higgs boson production at the LHC, Nucl. Phys. B 453 (1995) 17 [hep-ph/9504378] [INSPIRE].

[9] R. Harlander and P. Kant, Higgs production and decay: Analytic results at next-to-leading order QCD, JHEP 12 (2005) 015 [hep-ph/0509189] [INSPIRE].

[10] C. Anastasiou, S. Beerli, S. Bucherer, A. Daleo and Z. Kunszt, Two-loop amplitudes and master integrals for the production of a Higgs boson via a massive quark and a scalar-quark loop, JHEP 01 (2007) 082 [hep-ph/0611236] [INSPIRE].

[11] U. Aglietti, R. Bonciani, G. Degrassi and A. Vicini, Analytic Results for Virtual QCD Corrections to Higgs Production and Decay, JHEP 01 (2007) 021 [hep-ph/0611266] [INSPIRE].

[12] R. Bonciani, G. Degrassi and A. Vicini, Scalar particle contribution to Higgs production via gluon fusion at NLO, JHEP 11 (2007) 095 [arXiv:0709.4227] [INSPIRE].

[13] C. Anastasiou, C. Duhr, F. Dulat and B. Mistlberger, Soft triple-real radiation for Higgs production at N3LO, JHEP 07 (2013) 003 [arXiv:1302.4379] [INSPIRE].

[14] C. Anastasiou, C. Duhr, F. Dulat, F. Herzog and B. Mistlberger, Real-virtual contributions to the inclusive Higgs cross-section at $N^{3} L O$, JHEP 12 (2013) 088 [arXiv:1311.1425] [INSPIRE].

[15] C. Anastasiou, C. Duhr, F. Dulat, E. Furlan, T. Gehrmann, F. Herzog et al., Higgs Boson GluonFfusion Production Beyond Threshold in $N^{3} L O$ QCD, JHEP 03 (2015) 091 [arXiv: 1411.3584] [INSPIRE].

[16] Y. Li, A. von Manteuffel, R.M. Schabinger and H.X. Zhu, Soft-virtual corrections to Higgs production at $N^{3} L O$, Phys. Rev. D 91 (2015) 036008 [arXiv:1412.2771] [INSPIRE].

[17] C. Anastasiou, C. Duhr, F. Dulat, F. Herzog and B. Mistlberger, Higgs Boson Gluon-Fusion Production in QCD at Three Loops, Phys. Rev. Lett. 114 (2015) 212001 [arXiv:1503.06056] [INSPIRE].

[18] C. Anastasiou, C. Duhr, F. Dulat, E. Furlan, T. Gehrmann, F. Herzog et al., High precision determination of the gluon fusion Higgs boson cross-section at the LHC, JHEP 05 (2016) 058 [arXiv:1602.00695] [INSPIRE].

[19] R.V. Harlander and T. Neumann, Probing the nature of the Higgs-gluon coupling, Phys. Rev. D 88 (2013) 074015 [arXiv: 1308.2225] [INSPIRE]. 
[20] A. Banfi, A. Martin and V. Sanz, Probing top-partners in Higgs+jets, JHEP 08 (2014) 053 [arXiv: 1308.4771] [INSPIRE].

[21] A. Azatov and A. Paul, Probing Higgs couplings with high $p_{T}$ Higgs production, JHEP 01 (2014) 014 [arXiv: 1309.5273] [INSPIRE].

[22] C. Grojean, E. Salvioni, M. Schlaffer and A. Weiler, Very boosted Higgs in gluon fusion, JHEP 05 (2014) 022 [arXiv:1312.3317] [INSPIRE].

[23] M. Schlaffer, M. Spannowsky, M. Takeuchi, A. Weiler and C. Wymant, Boosted Higgs Shapes, Eur. Phys. J. C 74 (2014) 3120 [arXiv:1405.4295] [InSPIRE].

[24] M. Buschmann, C. Englert, D. Goncalves, T. Plehn and M. Spannowsky, Resolving the Higgs-Gluon Coupling with Jets, Phys. Rev. D 90 (2014) 013010 [arXiv:1405.7651] [INSPIRE].

[25] S. Dawson, I.M. Lewis and M. Zeng, Effective field theory for Higgs boson plus jet production, Phys. Rev. D 90 (2014) 093007 [arXiv:1409.6299] [INSPIRE].

[26] M. Buschmann, D. Goncalves, S. Kuttimalai, M. Schonherr, F. Krauss and T. Plehn, Mass Effects in the Higgs-Gluon Coupling: Boosted vs Off-Shell Production, JHEP 02 (2015) 038 [arXiv:1410.5806] [INSPIRE].

[27] D. Ghosh and M. Wiebusch, Dimension-six triple gluon operator in Higgs+jet observables, Phys. Rev. D 91 (2015) 031701 [arXiv:1411.2029] [INSPIRE].

[28] S. Dawson, I.M. Lewis and M. Zeng, Usefulness of effective field theory for boosted Higgs production, Phys. Rev. D 91 (2015) 074012 [arXiv:1501.04103] [INSPIRE].

[29] U. Langenegger, M. Spira and I. Strebel, Testing the Higgs Boson Coupling to Gluons, arXiv: 1507.01373 [INSPIRE].

[30] A. Azatov, C. Grojean, A. Paul and E. Salvioni, Resolving gluon fusion loops at current and future hadron colliders, JHEP 09 (2016) 123 [arXiv: 1608.00977] [INSPIRE].

[31] M. Grazzini, A. Ilnicka, M. Spira and M. Wiesemann, Modeling BSM effects on the Higgs transverse-momentum spectrum in an EFT approach, JHEP 03 (2017) 115 [arXiv: 1612.00283] [INSPIRE].

[32] R. Boughezal, F. Caola, K. Melnikov, F. Petriello and M. Schulze, Higgs boson production in association with a jet at next-to-next-to-leading order in perturbative QCD, JHEP 06 (2013) 072 [arXiv:1302.6216] [INSPIRE].

[33] X. Chen, T. Gehrmann, E.W.N. Glover and M. Jaquier, Precise QCD predictions for the production of Higgs + jet final states, Phys. Lett. B $\mathbf{7 4 0}$ (2015) 147 [arXiv:1408.5325] [INSPIRE].

[34] R. Boughezal, F. Caola, K. Melnikov, F. Petriello and M. Schulze, Higgs boson production in association with a jet at next-to-next-to-leading order, Phys. Rev. Lett. 115 (2015) 082003 [arXiv: 1504.07922] [INSPIRE].

[35] R. Boughezal, C. Focke, W. Giele, X. Liu and F. Petriello, Higgs boson production in association with a jet at NNLO using jettiness subtraction, Phys. Lett. B 748 (2015) 5 [arXiv: 1505. 03893] [INSPIRE].

[36] X. Chen, J. Cruz-Martinez, T. Gehrmann, E.W.N. Glover and M. Jaquier, NNLO QCD corrections to Higgs boson production at large transverse momentum, JHEP 10 (2016) 066 [arXiv: 1607.08817] [INSPIRE]. 
[37] M. Grazzini and H. Sargsyan, Heavy-quark mass effects in Higgs boson production at the LHC, JHEP 09 (2013) 129 [arXiv: 1306.4581] [INSPIRE].

[38] R.K. Ellis, I. Hinchliffe, M. Soldate and J.J. van der Bij, Higgs Decay to tau+ tau-: A Possible Signature of Intermediate Mass Higgs Bosons at the SSC, Nucl. Phys. B 297 (1988) 221 [inSPIRE].

[39] R.P. Kauffman, Higgs boson $p_{T}$ in gluon fusion, Phys. Rev. D 44 (1991) 1415 [inSPIRE].

[40] S.P. Jones, M. Kerner and G. Luisoni, Next-to-Leading-Order QCD Corrections to Higgs Boson Plus Jet Production with Full Top-Quark Mass Dependence, Phys. Rev. Lett. 120 (2018) 162001 [arXiv:1802.00349] [INSPIRE].

[41] J.M. Lindert, K. Kudashkin, K. Melnikov and C. Wever, Higgs bosons with large transverse momentum at the LHC, Phys. Lett. B 782 (2018) 210 [arXiv:1801.08226] [InSPIRE].

[42] T. Neumann, NLO Higgs+jet at Large Transverse Momenta Including Top Quark Mass Effects, J. Phys. Comm. 2 (2018) 095017 [arXiv: 1802.02981] [INSPIRE].

[43] K. Kudashkin, K. Melnikov and C. Wever, Two-loop amplitudes for processes $g g \rightarrow H g, q g \rightarrow H q$ and $q \bar{q} \rightarrow H g$ at large Higgs transverse momentum, JHEP 02 (2018) 135 [arXiv: 1712.06549] [INSPIRE].

[44] J.M. Lindert, K. Melnikov, L. Tancredi and C. Wever, Top-bottom interference effects in Higgs plus jet production at the LHC, Phys. Rev. Lett. 118 (2017) 252002 [arXiv: 1703.03886] [INSPIRE].

[45] F. Caola, J.M. Lindert, K. Melnikov, P.F. Monni, L. Tancredi and C. Wever, Bottom-quark effects in Higgs production at intermediate transverse momentum, JHEP 09 (2018) 035 [arXiv: 1804.07632] [INSPIRE].

[46] K. Melnikov, L. Tancredi and C. Wever, Two-loop gg $\rightarrow$ Hg amplitude mediated by a nearly massless quark, JHEP 11 (2016) 104 [arXiv:1610.03747] [INSPIRE].

[47] K. Melnikov, L. Tancredi and C. Wever, Two-loop amplitudes for $q g \rightarrow H q$ and $q \bar{q} \rightarrow H g$ mediated by a nearly massless quark, Phys. Rev. D 95 (2017) 054012 [arXiv:1702.00426] [INSPIRE].

[48] R. Bonciani, V. Del Duca, H. Frellesvig, J.M. Henn, F. Moriello and V.A. Smirnov, Two-loop planar master integrals for Higgs $\rightarrow 3$ partons with full heavy-quark mass dependence, JHEP 12 (2016) 096 [arXiv: 1609.06685] [INSPIRE].

[49] U. Aglietti, R. Bonciani, G. Degrassi and A. Vicini, Two loop light fermion contribution to Higgs production and decays, Phys. Lett. B 595 (2004) 432 [hep-ph/0404071] [INSPIRE].

[50] U. Aglietti, R. Bonciani, G. Degrassi and A. Vicini, Master integrals for the two-loop light fermion contributions to $g g \rightarrow H$ and $H \rightarrow \gamma \gamma$, Phys. Lett. B 600 (2004) 57 [hep-ph/0407162] [INSPIRE].

[51] G. Degrassi and F. Maltoni, Two-loop electroweak corrections to Higgs production at hadron colliders, Phys. Lett. B 600 (2004) 255 [hep-ph/0407249] [INSPIRE].

[52] G. Degrassi and F. Maltoni, Two-loop electroweak corrections to the Higgs-boson decay $H \rightarrow \gamma \gamma$, Nucl. Phys. B 724 (2005) 183 [hep-ph/0504137] [INSPIRE].

[53] S. Actis, G. Passarino, C. Sturm and S. Uccirati, NLO Electroweak Corrections to Higgs Boson Production at Hadron Colliders, Phys. Lett. B 670 (2008) 12 [arXiv:0809.1301] [INSPIRE]. 
[54] M. Bonetti, K. Melnikov and L. Tancredi, Two-loop electroweak corrections to Higgs-gluon couplings to higher orders in the dimensional regularization parameter, Nucl. Phys. B 916 (2017) 709 [arXiv: 1610.05497] [INSPIRE].

[55] M. Bonetti, K. Melnikov and L. Tancredi, Three-loop mixed QCD-electroweak corrections to Higgs boson gluon fusion, Phys. Rev. D 97 (2018) 034004 [arXiv:1711.11113] [INSPIRE].

[56] C. Anastasiou, R. Boughezal and F. Petriello, Mixed QCD-electroweak corrections to Higgs boson production in gluon fusion, JHEP 04 (2009) 003 [arXiv:0811.3458] [INSPIRE].

[57] M. Bonetti, K. Melnikov and L. Tancredi, Higher order corrections to mixed QCD-EW contributions to Higgs boson production in gluon fusion, Phys. Rev. D 97 (2018) 056017 [Erratum ibid. D 97 (2018) 099906] [arXiv:1801.10403] [INSPIRE].

[58] A.V. Smirnov, FIRE5: a C++ implementation of Feynman Integral REduction, Comput. Phys. Commun. 189 (2015) 182 [arXiv:1408.2372] [INSPIRE].

[59] R.N. Lee, LiteRed 1.4: a powerful tool for reduction of multiloop integrals, J. Phys. Conf. Ser. 523 (2014) 012059 [arXiv: 1310.1145] [InSPIRE].

[60] C. Anastasiou and A. Lazopoulos, Automatic integral reduction for higher order perturbative calculations, JHEP 07 (2004) 046 [hep-ph/0404258] [INSPIRE].

[61] A. von Manteuffel and C. Studerus, Reduze 2 - Distributed Feynman Integral Reduction, arXiv: 1201.4330 [INSPIRE].

[62] P. Maierhöfer, J. Usovitsch and P. Uwer, Kira - A Feynman integral reduction program, Comput. Phys. Commun. 230 (2018) 99 [arXiv:1705.05610] [INSPIRE].

[63] K.G. Chetyrkin and F.V. Tkachov, Integration by Parts: The Algorithm to Calculate $\beta$-functions in 4 Loops, Nucl. Phys. B 192 (1981) 159 [InSPIRE].

[64] F.V. Tkachov, A Theorem on Analytical Calculability of Four Loop Renormalization Group Functions, Phys. Lett. 100B (1981) 65 [INSPIRE].

[65] S. Laporta, High precision calculation of multiloop Feynman integrals by difference equations, Int. J. Mod. Phys. A 15 (2000) 5087 [hep-ph/0102033] [InSPIRE].

[66] T. Gehrmann and E. Remiddi, Differential equations for two loop four point functions, Nucl. Phys. B 580 (2000) 485 [hep-ph/9912329] [INSPIRE].

[67] A.V. Kotikov, Differential equations method: New technique for massive Feynman diagrams calculation, Phys. Lett. B 254 (1991) 158 [INSPIRE].

[68] E. Remiddi, Differential equations for Feynman graph amplitudes, Nuovo Cim. A 110 (1997) 1435 [hep-th/9711188] [INSPIRE].

[69] T. Gehrmann and E. Remiddi, Analytic continuation of massless two loop four point functions, Nucl. Phys. B 640 (2002) 379 [hep-ph/0207020] [INSPIRE].

[70] M. Argeri and P. Mastrolia, Feynman Diagrams and Differential Equations, Int. J. Mod. Phys. A 22 (2007) 4375 [arXiv:0707.4037] [INSPIRE].

[71] J.M. Henn, Lectures on differential equations for Feynman integrals, J. Phys. A 48 (2015) 153001 [arXiv:1412.2296] [INSPIRE].

[72] J.M. Henn, Multiloop integrals in dimensional regularization made simple, Phys. Rev. Lett. 110 (2013) 251601 [arXiv:1304.1806] [INSPIRE]. 
[73] M. Argeri, S. Di Vita, P. Mastrolia, E. Mirabella, J. Schlenk, U. Schubert et al., Magnus and Dyson Series for Master Integrals, JHEP 03 (2014) 082 [arXiv:1401.2979] [InSPIRE].

[74] R.N. Lee, Reducing differential equations for multiloop master integrals, JHEP 04 (2015) 108 [arXiv: 1411.0911] [INSPIRE].

[75] A. Georgoudis, K.J. Larsen and Y. Zhang, Azurite: An algebraic geometry based package for finding bases of loop integrals, Comput. Phys. Commun. 221 (2017) 203 [arXiv:1612.04252] [INSPIRE].

[76] T. Gehrmann, A. von Manteuffel, L. Tancredi and E. Weihs, The two-loop master integrals for $q \bar{q} \rightarrow V V$, JHEP 06 (2014) 032 [arXiv: 1404.4853] [INSPIRE].

[77] M. Becchetti and R. Bonciani, Two-Loop Master Integrals for the Planar QCD Massive Corrections to Di-photon and Di-jet Hadro-production, JHEP 01 (2018) 048 [arXiv: 1712.02537] [INSPIRE].

[78] K.-T. Chen, Iterated path integrals, Bull. Am. Math. Soc. 83 (1977) 831.

[79] A. Goncharov, Polylogarithms in arithmetic and geometry, Proceedings of the International Congress of Mathematicians 1,2 (1995) 374.

[80] A.B. Goncharov, Multiple polylogarithms and mixed Tate motives, math/0103059 [INSPIRE].

[81] E. Remiddi and J.A.M. Vermaseren, Harmonic polylogarithms, Int. J. Mod. Phys. A 15 (2000) 725 [hep-ph/9905237] [INSPIRE].

[82] C.W. Bauer, A. Frink and R. Kreckel, Introduction to the GiNaC framework for symbolic computation within the C++ programming language, J. Symb. Comput. 33 (2000) 1 [cs/0004015].

[83] J. Vollinga and S. Weinzierl, Numerical evaluation of multiple polylogarithms, Comput. Phys. Commun. 167 (2005) 177 [hep-ph/0410259] [INSPIRE].

[84] A.V. Smirnov, FIESTA4: Optimized Feynman integral calculations with GPU support, Comput. Phys. Commun. 204 (2016) 189 [arXiv:1511.03614] [INSPIRE].

[85] U. Aglietti and R. Bonciani, Master integrals with one massive propagator for the two loop electroweak form-factor, Nucl. Phys. B 668 (2003) 3 [hep-ph/0304028] [INSPIRE].

[86] U. Aglietti and R. Bonciani, Master integrals with 2 and 3 massive propagators for the 2 loop electroweak form-factor - planar case, Nucl. Phys. B 698 (2004) 277 [hep-ph/0401193] [INSPIRE].

[87] A.B. Goncharov, Multiple polylogarithms and mixed Tate motives, math/0103059 [INSPIRE].

[88] A.B. Goncharov, M. Spradlin, C. Vergu and A. Volovich, Classical Polylogarithms for Amplitudes and Wilson Loops, Phys. Rev. Lett. 105 (2010) 151605 [arXiv:1006.5703] [INSPIRE].

[89] C. Duhr, H. Gangl and J.R. Rhodes, From polygons and symbols to polylogarithmic functions, JHEP 10 (2012) 075 [arXiv:1110.0458] [INSPIRE]. 